\title{
INVERSE PROBLEMS FOR MULTIPLE INVARIANT CURVES
}

\author{
COLIN CHRISTOPHER ${ }^{1}$, JAUME LLIBRE $^{2}$, CHARA PANTAZI $^{3}$ AND \\ SEBASTIAN WALCHER ${ }^{4}$
}

\begin{abstract}
Planar polynomial vector fields which admit invariant algebraic curves, Darboux integrating factors or Darboux first integrals are of special interest. In the present paper we solve the inverse problem for invariant algebraic curves with a given multiplicity and for integrating factors, under generic assumptions regarding the (multiple) invariant algebraic curves involved. In particular we prove, in this generic scenario, that the existence of a Darboux integrating factor implies Darboux integrability. Furthermore we construct examples where the genericity assumption does not hold and indicate that the situation is different for these.
\end{abstract}

\section{INTRODUCTION}

In 1878, Darboux [8] published his original work on the integrability of polynomial differential equations in the plane. He showed how the existence of sufficiently many invariant algebraic curves forces the integrability of a polynomial system. Darboux's idea was to use the invariant algebraic curves $\left\{f_{i}=0\right\}$ of the system for constructing an integrating factor of the form

$$
\prod_{i=1}^{r} f_{i}^{l_{i}} .
$$

It was shown in [12] that such integrating factors account for all polynomial differential systems with elementary first integrals. (For precise definitions and basic results on the Darboux theory see Section 2).

1991 Mathematics Subject Classification. Primary 34C05, 34A34, 34C14.

Key words and phrases. polynomial differential system, invariant algebraic curve, integrating factor, exponential factor, multiplicity, Darboux integrability.

The second and third authors are partially supported by a MCYT grant MTM 200506098-C02-01 and by a CIRIT grant number 2001SGR 00550. The first and fourth authors acknowledge the support of the Mathematics Department and the CRM at Universitat Autònoma de Barcelona during visits when this manuscript was prepared. 
Darboux's integrability theory has been extended taking into account the multiplicity or coalescence of invariant algebraic curves through the existence of exponential factors, see $[2,4,5,7]$. Thus the Darboux integrating factors are extended into the form

$$
\prod_{i=1}^{r} f_{i}^{l_{i}} \prod_{j=1}^{s} \exp \left(g_{j} / h_{j}\right)
$$

where the functions $\exp \left(g_{i} / h_{i}\right)$ (i.e. the exponential factors) satisfy an equation similar to the $f_{i}$ defining the invariant algebraic curves. Such functions are called Darboux. It has been shown by Singer [14] (with the additional comments of [3] for example) that polynomial differential systems with Darboux integrating factors are exactly those with Liouvillian first integrals.

The paper [7] contains, among other results, a study of a kind of inverse problem in the Darboux theory of integrability. More precisely, assuming certain algebraic conditions, [7] contains an explicit basis of the polynomial vector fields $X$ which have a given set of algebraic curves with a given multiplicity as invariant curves for their flow. Clearly, the study of such inverse problems can also greatly increase our knowledge of the direct applications of Darboux integrability.

In this paper, we wish to study this inverse problem in greater depth and detail, and show that this does indeed lead us to a new understanding of the consequences of Darboux integrability.

In Section 3, assuming certain (generic) geometric conditions on the curves, we give precise bounds for the degrees of the polynomials in the defining equations of polynomial vector fields with given collections of multiple invariant algebraic curves (Theorems 6 and 9). We also refer to Section 3 for a precise definition of the multiplicity of an invariant algebraic curve.

In Section 4, we study another type of "inverse problem" in that we seek polynomial vector fields which have a given Darboux function as a first integral or integrating factor. Such studies have been carried out before $[10,15,16]$. Here, we extend the study to multiple curves. In Theorem 12 we show, under generic conditions, that a polynomial vector field with a Darboux inverse integrating factor must admit a Darboux first integral.

Finally, in Section 5 we construct several examples, to show that the results of the previous sections do not hold without the imposition of some genericity assumptions.

Our approach is mainly algebraic and formal. A different (analytictopological) approach to some of these questions is given by Żoładek in [17]. Let us briefly sketch this for curves of multiplicity one. Suppose that $D$ is a Darboux integrating factor, without exponential terms, for the differential 
equation (1) below. Then

$$
\phi=\int D(P d y-Q d x)
$$

defines a multi-valued integral outside the set $Z$ of zeros of the curves $\left\{f_{i}=0\right\}$. The effect of passing around a non-trivial loop $\gamma$ in the complement of $Z$ takes $\phi$ to $h_{\gamma}(\phi)=a_{\gamma} \phi+b_{\gamma}$ for some constants $a_{\gamma}$ and $b_{\gamma}$. Under the conditions of Theorem 10 (all the curves being non-multiple) the set $Z$ together with the line at infinity form a normal crossing divisor of $\mathbb{P}^{2}$, and it is well-known that in this case its complement has an abelian fundamental group. This means that the maps $h_{\gamma}$ commute. It is then straightforward to show that either $a_{\gamma}=1$ for all $\gamma$ or the addition of a constant to $\phi$ makes all the $b_{\gamma}$ vanish. In the first case, $\phi-\sum c_{i} \log \left(f_{i}\right)$ is single valued for some constants $c_{i}$ determined by the $b_{\gamma}$, and in the second, $\phi / \prod f_{i}^{m_{i}}$ is single valued for some constants $m_{i}$ determined by the $a_{\gamma}$. Growth estimates show that these functions are rational and hence one obtains either $\exp (\phi)$ or $\phi$ as a Darboux first integral. When $D$ contains exponential terms (or equivalently, algebraic curves with multiplicity greater than one), it is necessary to discuss the extended monodromy group in Żołądek's approach, which seems to be more difficult to understand and to work with.

An algebraic approach to questions of Darboux integrability was given in [6] in the case of curves of multiplicity one; and extended to curves of multiplicity greater than one in [7], and is further extended here. It works on a more elementary and explicit level, and seems to be more appropriate for concrete computations. It also allows the clarification of the geometric nondegeneracy conditions which underly the theorems; see Theorem 10 and Section 4. Moreover, algebraic tools are naturally suited for the (counter)examples in the final section. Thus it seems reasonable and useful to pursue the algebraic approach in its own right.

\section{BASIC NOTIONS}

We consider planar polynomial differential systems of the form

$$
\frac{d x}{d t}=\dot{x}=P(x, y), \quad \frac{d y}{d t}=\dot{y}=Q(x, y),
$$

where $P, Q \in \mathbb{C}[x, y]$ and $t \in \mathbb{C}$ (though, in applications we might wish to restrict to only real values). We associate to the polynomial differential system (1) in $\mathbb{C}^{2}$ the polynomial vector field

$$
X=P(x, y) \frac{\partial}{\partial x}+Q(x, y) \frac{\partial}{\partial y} .
$$


Sometimes, the polynomial vector field $X$ will be denoted simply by $(P, Q)$.

The degree $m$ of the polynomial differential system (1) or of the polynomial vector field $X$ will be denoted by $\delta X$ and is the maximum of the degrees of the polynomials $P$ and $Q$. The degree of a polynomial $P$ is denoted by $\delta P$. The degree of a rational function $P / Q$ is defined as $\delta(P / Q)=\max \{\delta P, \delta Q\}$.

A first integral of system (1) on an open subset $U$ of $\mathbb{C}^{2}$ is an analytic function $H: U \rightarrow \mathbb{C}$ which is not constant on any connected component of $U$ but constant on every solution curve $(x(t), y(t))$ of $(1)$ in $U$. We say that the polynomial system (1) is integrable on $U$ if there is a first integral on $U$. Equivalently, we seek a function $H$ on $U$ such that $X(H)$ vanishes identically. This latter version is more useful, as it is easier to work with algebraically.

As usual, for an analytic function $f$ we denote by $f_{x}$ and $f_{y}$ the partial derivatives with respect to $x$ and $y$, respectively. The Hamiltonian vector field $X_{f}$ is defined by

$$
X_{f}=-f_{y} \frac{\partial}{\partial x}+f_{x} \frac{\partial}{\partial y}
$$

and clearly admits $f$ as a first integral.

An analytic function $R: U \rightarrow \mathbb{C}$ which is not identically zero on $U$ is called an integrating factor of system (1) if it satisfies

$$
X(R)=-R \cdot \operatorname{div}(X)
$$

in $U$. As usual the divergence of the vector field $X$ is defined by $\operatorname{div}(X)=$ $\frac{\partial P}{\partial x}+\frac{\partial Q}{\partial y}$. If $R: U \rightarrow \mathbb{C}$ is an integrating factor of system (1) and $W=$ $U \backslash\{R=0\}$ then we call $V=1 / R: W \rightarrow \mathbb{C}$ an inverse integrating factor of (1).

We are interested in invariant algebraic sets for polynomial vector fields. The following result is well-known, but we include it for completeness.

Lemma 1. Let $X$ be a polynomial vector field and $f_{1}, \cdots, f_{r}$ polynomials.

(a) If $X\left(f_{i}\right) \in\left\langle f_{1}, \cdots, f_{r}\right\rangle$ for all $i$ then the common zero set $Z$ of $f_{1}, \cdots, f_{r}$ is invariant for the local flow of $X$.

(b) Conversely, if the common zero set of $f_{1} \cdots, f_{r}$ is invariant for the local flow of $X$ then $X\left(f_{i}\right) \in \operatorname{rad}\left\langle f_{1}, \cdots, f_{r}\right\rangle$ for all $i$, and in particular $X\left(f_{i}\right) \in\left\langle f_{1}, \cdots, f_{r}\right\rangle$ for all $i$ if the ideal is radical. that

Proof: Since $X\left(f_{i}\right) \in\left\langle f_{1}, \cdots, f_{r}\right\rangle$ for all $i$ there are $W_{i j} \in \mathbb{C}[x, y]$ such

$$
X\left(f_{i}\right)=\sum_{j=1}^{r} W_{i j} f_{j}
$$


Let $v(t)$ be a solution of the differential equation such that $v(0) \in Z$. Since

$$
\frac{d}{d t} f_{i}(v(t))=X\left(f_{i}\right)(v(t))=\sum_{j=1}^{r} W_{i j}(v(t)) f_{j}(v(t))
$$

the $f_{i}(v(t))$ satisfy a homogeneous linear system of differential equations with coefficient matrix $\left(W_{i j}(v(t))\right)_{i, j}$, and initial value zero. By uniqueness, the $f_{i}(v(t))$ are identically zero, and thus $v(t) \in Z$.

(b) Conversely, let $v(t)$ be a solution contained in $Z$, and $1 \leq i \leq r$. Then,

$$
0=\frac{d}{d t} f_{i}(v(t))=X\left(f_{i}\right)(v(t))
$$

shows $X\left(f_{i}\right)(v(0))=0$ and therefore $X\left(f_{i}\right)$ vanishes on $Z$. By the Hilbert Nullstellensatz (see for instance [13]) we have $X\left(f_{i}\right) \in \operatorname{rad}\left\langle f_{1}, \cdots, f_{r}\right\rangle$, and this ideal equals $\left\langle f_{1}, \cdots, f_{r}\right\rangle$ if the latter is radical.

In particular, let $f \in \mathbb{C}[x, y]$ be an irreducible polynomial. The algebraic curve $f(x, y)=0$ is an invariant algebraic curve of the polynomial vector field $X$ if and only if for some polynomial $K \in \mathbb{C}[x, y]$ we have

$$
X(f)=P \frac{\partial f}{\partial x}+Q \frac{\partial f}{\partial y}=K \cdot f .
$$

The polynomial $K$ is called the cofactor of the invariant algebraic curve $f=0$. If the polynomial vector field has degree $m$, any cofactor must have degree at most $m-1$.

The following consequence of Lemma 1 will be useful for constructions later on.

Lemma 2. Let $X$ be a planar polynomial vector field, and let $f_{0}, f_{1}$ be relatively prime polynomials. Moreover, assume that $X\left(f_{0}\right)=L_{0} f_{0}$ for some polynomial $L_{0}$. Then, $X\left(f_{1}\right)=L_{2} f_{1}+L_{1} f_{0}$ for suitable polynomials $L_{1}, L_{2}$ only if every point of the intersection of $\left\{f_{0}=0\right\}$ and $\left\{f_{1}=0\right\}$ is a critical point of $X$.

In addition, if all intersections of $\left\{f_{0}=0\right\}$ and $\left\{f_{1}=0\right\}$ are transversal (i.e. the derivatives are linearly independent at all intersection points) then the condition is also sufficient.

Proof: The first assumption is a direct consequence of Lemma 1, since the connected components of $\left\{f_{0}=0\right\} \bigcap\left\{f_{1}=0\right\}$ are points, due to relative primeness. For the second assertion, recall that the transversality condition holds if and only if the ideal $\left\langle f_{0}, f_{1}\right\rangle$ is radical. (This may be proven using the Chinese remainder theorem: The ideal is radical if and only if $\mathbb{C}[x, y] /\left\langle f_{0}, f_{1}\right\rangle$ is a direct sum of fields. See the arguments in [15], for instance.) 
If two invariant algebraic curves coalesce then we obtain in a natural way the notion of exponential factors, see [2] and [7].

Let $g, h \in \mathbb{C}[x, y]$ and assume that $g$ and $h$ are relatively prime. Then the function $\exp (g / h)$ is called an exponential factor of the polynomial vector field $X$ if for some polynomial $L \in \mathbb{C}[x, y]$,

$$
X\left(\exp \left(\frac{g}{h}\right)\right)=L \cdot \exp \left(\frac{g}{h}\right) .
$$

As above we call $L$ the cofactor of the exponential factor $\exp (g / h)$. From [2] the following result is known.

Proposition 3. If $\exp (g / h)$ is an exponential factor for the polynomial vector field $X$ then $h=0$ defines an invariant algebraic curve of $X$.

The next result is due essentially to Darboux (at least in the case without the exponential factors), see [8].

Theorem 4. Suppose that a polynomial vector field $X$ admits $p$ irreducible invariant algebraic curves $\left\{f_{i}=0\right\}$ with cofactors $K_{i}$ for $i=1, \ldots, p$ and $q$ exponential factors $\exp \left(g_{j} / h_{j}\right)$ with cofactors $L_{j}$ for $j=1, \ldots, q$.

(a) Then there exist $\lambda_{i}, \mu_{j} \in \mathbb{C}$ not all zero such that

$$
\sum_{i=1}^{p} \lambda_{i} K_{i}+\sum_{j=1}^{q} \mu_{j} L_{j}=\operatorname{div}(X),
$$

if and only if the (multi-valued) function

$$
f_{1}^{\lambda_{1}} \cdots f_{p}^{\lambda_{p}}\left(\exp \left(\frac{g_{1}}{h_{1}}\right)\right)^{\mu_{1}} \cdots\left(\exp \left(\frac{g_{q}}{h_{q}}\right)\right)^{\mu_{q}},
$$

is an inverse integrating factor of $X$.

(b) There exist $\lambda_{i}, \mu_{j} \in \mathbb{C}$ not all zero such that

$$
\sum_{i=1}^{p} \lambda_{i} K_{i}+\sum_{j=1}^{q} \mu_{j} L_{j}=0
$$

if and only if the function given in (4) is a first integral of $X$.

The precise relationship between exponential factors and multiple invariant algebraic curves will be discussed in the following section.

\section{Generalized invariant Algebraic CURVES}

We refer to [7] for more details about the contents of this section. However, we have altered the notation slightly in order to facilitate the exposition here.

Consider a polynomial vector field $X$, with an invariant algebraic curve $\left\{f_{0}=0\right\}$. We shall always assume in this section that $f_{0}$ is irreducible as 
a polynomial. We say that a polynomial $f_{0} \in \mathbb{C}[x, y]$ admits a generalized invariant algebraic curve of order $n$ based on $\left\{f_{0}=0\right\}$ if there are polynomials $f_{1}, \cdots, f_{n-1}$ with $\delta f_{i} \leq \delta f_{0}$, and $L_{0}, L_{1}, \cdots, L_{n-1} \in \mathbb{C}[x, y]$ such that

$$
\begin{aligned}
X\left(f_{0}\right) & =L_{0} f_{0}, \\
X\left(f_{1}\right) & =L_{0} f_{1}+L_{1} f_{0}, \\
& \vdots \\
X\left(f_{n-1}\right) & =L_{0} f_{n-1}+L_{1} f_{n-2}+\cdots+L_{n-1} f_{0} .
\end{aligned}
$$

The condition (5) can be more helpfully represented by the use of matrices as follows. We let $\varepsilon$ be the $n \times n$ matrix

$$
\varepsilon=\left(\begin{array}{ccccc}
0 & 1 & 0 & \cdots & 0 \\
& 0 & 1 & & \vdots \\
\vdots & & \ddots & & 0 \\
& & & 0 & 1 \\
0 & & \cdots & & 0
\end{array}\right)
$$

and represent the generalized invariant algebraic curve by a matrix $F$ given by

$$
F=f_{0}+\varepsilon f_{1}+\cdots+f_{n-1} \varepsilon^{n-1}=\left(\begin{array}{ccccl}
f_{0} & f_{1} & f_{2} & \cdots & f_{n-1} \\
0 & f_{0} & f_{1} & \cdots & f_{n-2} \\
\vdots & \ddots & \ddots & & \vdots \\
\vdots & & \ddots & f_{0} & f_{1} \\
0 & \cdots & \cdots & 0 & f_{0}
\end{array}\right) .
$$

Then condition (5) becomes

$$
X(B)=F L,
$$

where,

$$
B=\left(\begin{array}{l}
f_{n-1} \\
\vdots \\
\vdots \\
f_{0}
\end{array}\right), \quad L=\left(\begin{array}{l}
L_{n-1} \\
\vdots \\
\vdots \\
L_{0}
\end{array}\right)
$$

The generalized curve is said to be nondegenerate if $f_{1}$ is not a multiple of $f_{0}$. Degenerate generalized invariant algebraic curves are of little interest as they can always be recast as non-degenerate ones (possibly of lower order) unless they are multiples of simple curves (i.e. just $k(\epsilon) f_{0}$, for some polynomial $k$ ). From now on we will always assume nondegeneracy. 
The multiplicity of $f_{0}=0$ with respect to $X$ is the maximal order of all nondegenerate generalized algebraic curves based on $f_{0}=0$. Other notions of multiplicity and their interrelations are discussed in [7].

Generalized invariant algebraic curves occur naturally when one considers exponential factors. Using relation (6) we get

$$
\begin{aligned}
\log (F) & =\log \left(f_{0}\right)+\varepsilon \frac{f_{1}}{f_{0}}+\varepsilon^{2}\left(\frac{f_{2}}{f_{0}}-\frac{f_{1}^{2}}{2 f_{0}^{2}}\right)+\cdots \\
& =F_{0}+\varepsilon F_{1}+\varepsilon^{2} F_{2}+\cdots+\varepsilon^{n-1} F_{n-1},
\end{aligned}
$$

where $F_{0}=\log \left(f_{0}\right)$ and the $F_{i}$ are rational functions in $x$ and $y$ of degree at most $k i$ for $i \geq 1$. It is easy to show that the expressions $\exp \left(F_{i}\right)$ are exponential factors of the vector field $X$ with cofactors $L_{i}$ for $i=1, \cdots, n-$ 1. Thus, a generalized invariant algebraic curve of order $n$ gives rise to $n-1$ exponential factors $\exp \left(g_{j} / f_{0}^{j}\right)$ for $1 \leq j \leq n-1$, with $g_{i}$ coprime to $f_{0}$. Conversely, exponential factors give rise to generalized invariant algebraic curves. Once again, more details can be found in [7].

The main topic of the present paper is the inverse problem for generalized and multiple invariant algebraic curves, and applications to Darboux integrals and integrating factors. Thus, given generalized invariant algebraic curves $F^{(i)}$, we wish to determine all polynomial vector fields $X$ such that relations $(7)$ hold for suitable vectors $L^{(i)}$. A solution of the inverse problem for generalized invariant algebraic curves was given in [7], Theorem 5.11. We will improve this theorem to obtain bounds on the degrees of the arbitrary polynomials which appear in the solution. This will form an essential part of our proof of the main result about Darboux integrals and integrating factors. Moreover, we will explore the meaning of the ideal $I$ introduced in [7], and show that it is trivial under some natural genericity conditions, answering a question posed in that paper.

We first consider the case of a single generalized invariant algebraic curve

$$
F=f_{0}+\varepsilon f_{1}+\cdots+\varepsilon^{n-1} f_{n-1}
$$

based on a curve $\left\{f_{0}=0\right\}$ of degree $k$ and order $n$ in a polynomial vector field $X$, so that (7) holds for some appropriate matrix $L$.

We introduce the notation

$$
X_{F}=\left(\begin{array}{l}
X_{f_{n-1}} \\
\vdots \\
\vdots \\
X_{f_{0}}
\end{array}\right), \quad X_{F^{\prime}}=\left(\begin{array}{l}
X_{F_{n-1}} \\
\vdots \\
\vdots \\
X_{F_{0}}
\end{array}\right), \quad B^{\prime}=\left(\begin{array}{l}
F_{n-1} \\
\vdots \\
\vdots \\
F_{0}
\end{array}\right) \text {, }
$$

where $X_{f}$ is the Hamiltonian vector field described in (3). Thus $X_{F}$ and $X_{F^{\prime}}$ are vectors of operators. 
We denote by $|F|$ the determinant of the matrix $F$. From (6) we find $|F|=f_{0}^{n}$. We also denote by $F^{a}=|F| F^{-1}$ the adjoint matrix of $F$.

The following result appears in Theorem 5.12 [7], where the authors characterize the polynomial vector fields which admit given generalized invariant curves $F$, and thus solve the inverse problem in this case. We denote the Poisson bracket of two functions $f(x, y)$ and $g(x, y)$ by

$$
\{f, g\}=f_{x} g_{y}-f_{y} g_{x} .
$$

We also denote by $I$ the ideal generated in $\mathbb{C}[x, y]$ by $|F|,|F| F_{i x},|F| F_{i y}$ and $|F|\left\{F_{i}, F_{j}\right\}$ for $i, j=0, \cdots, n-1$. It is a non-trivial result of [7] that each of these terms is a polynomial.

Theorem 5. Let $I$ be as above, then for any $h \in I$, all polynomial vector field $X$ admitting $F$ as a generalized invariant algebraic curve of order $n$ can be written in the form

$$
h X=|F| X_{0}+C^{T} F^{a} X_{F}=|F| X_{0}+C^{T}|F| X_{F^{\prime}},
$$

with $X_{0}$ an arbitrary polynomial vector field and $C=\left(C_{n-1}, \cdots, C_{0}\right)^{T}$ a vector of arbitrary polynomials $C_{i} \in \mathbb{C}[x, y]$. In particular, if $I=\langle 1\rangle$, we can write $X$ in the form

$$
X=|F| X_{0}+C^{T} F^{a} X_{F}=|F| X_{0}+C^{T}|F| X_{F^{\prime}} .
$$

Theorem 5 is a generalization of [6], Theorem 1; see also [15] for vector fields with one invariant algebraic curve. This theorem does not provide information on the degrees of the arbitrary polynomials which appear in (10). In the following theorem, given additional conditions, we determine degree bounds for these. These bounds will be an essential point in the analysis in the following section.

Theorem 6. Let $f_{0}=0$ be an invariant algebraic curve of multiplicity $n$ with $\delta f_{0}=k$ and let $F$ be its associated matrix (6). Assume that the following hold.

(i) $I=\langle 1\rangle$,

(ii) $f_{0}$ has distinct factors in the highest order terms; and

(iii) $f_{0}$ and $f_{1}$ have no common factors in the highest order terms.

Then all the polynomial vector fields $X$ having $F$ as a multiple invariant algebraic curve can be written in the form (10) with $\delta X_{0} \leq \delta X-n k$ and $\delta C_{i} \leq \delta X-n k+1$ for $i=0, \cdots, n-1$.

As a preliminary step before the proof of this result, we state and prove an auxiliary result. We denote by $f^{*}$ the terms of highest degree of the polynomial $f \in \mathbb{C}[x, y]$. Moreover, $F^{*}$ is determined for $F$ in (6) by replacing 
$f_{i}$ by $f_{i}^{*}$ for $i=0, \cdots, n-1$; and in a similar way we define $C^{*}, X_{0}^{*}$ and $X_{F^{*}}$.

Lemma 7. Let $f_{0}=0$ define an invariant algebraic curve of multiplicity $n$ for $X$, and let $F$ be given by (6), and $C, X_{0}$ and $X_{F}$ as in (10). If $f_{0}$ satisfies the assumptions of Theorem 6 and the equality

$$
\left|F^{*}\right| X_{0}^{*}+C^{* T} F^{* a} X_{F^{*}}=0
$$

holds, then there is $n \times 1$ matrix $\bar{C}$ such that $C^{* T}=\bar{C}^{* T} F^{*}$ and $X_{0}^{*}=$ $-\bar{C} X_{F^{*}}$.

Proof: (i) For $n>1$ we have

$$
F^{*}=f_{0}^{*}\left(I_{n}+\frac{f_{1}^{*}}{f_{0}^{*}} \varepsilon+\cdots+\frac{f_{n-1}^{*}}{f_{0}^{*}} \varepsilon^{n-1}\right)=f_{0}^{*}\left(I_{n}+N\right),
$$

and hence we get

$$
F^{*-1}=f_{0}^{*-1}\left(I_{n}-N+N^{2}-\cdots+(-1)^{n-1} N^{n-1}\right) .
$$

Therefore, the only entry of $F^{* a}=f_{0}^{* n} F^{*-1}$ which is not a multiple of $f_{0}^{*}$ is the upper right one, since the contributions of $N, \cdots, N^{n-2}$ provide only multiples of $f_{0}^{*}$, and the only nonzero entry of $N^{n-1}$ is $\left(f_{1}^{*} / f_{0}^{*}\right)^{n-1}$, in the upper right position. Hence, the unique element in $C^{* T} F^{* a} X_{F^{*}}$ that is not automatically divisible by $f_{0}^{*}$ is $C_{n-1}^{*} f_{1}^{* n-1} X_{f_{0}^{*}}$.

(ii) After this technical preparation, we claim that $C^{* T}=\bar{C}^{* T} F^{*}$ for some $n \times 1$ matrix $\bar{C}$. For $n=1$ we have

$$
f_{0}^{*} X_{0}^{*}+C^{*} X_{f_{0}^{*}}=0 .
$$

Since $f_{0}^{*}$ has no multiple prime factors, no prime factor of $f_{0}^{*}$ divides both entries of $X_{f_{0}^{*}}$, and $C^{*} \in\left\langle f_{0}^{*}\right\rangle$ follows. Proceed by induction. Condition (11), together with (i), implies that $f_{0}^{*} \operatorname{divides} C_{n-1}^{*} f_{1}^{* n-1} X_{f_{0}^{*}}$. Since $f_{0}^{*}$ has no multiple factors, and $f_{0}^{*}$ and $f_{1}^{*}$ are relatively prime, we find that $f_{0}^{*}$ divides $C_{n-1}^{*}$. We may therefore write

$$
\left(C_{n-1}^{*}, C_{n-2}^{*}, \cdots, C_{0}^{*}\right)=\left(\tilde{C}_{n-1}, \tilde{C}_{n-2}, \cdots, \tilde{C}_{0}\right)\left(\begin{array}{llll}
f_{0}^{*} & f_{1}^{*} & \cdots & f_{n-1}^{*} \\
0 & & I_{n-1} &
\end{array}\right),
$$

for some polynomials $\tilde{C}_{n-1}, \cdots, \tilde{C}_{0}$. Substituting this in (11), we obtain

$$
\begin{aligned}
0 & =f_{0}^{* n} X_{0}^{*}+\tilde{C}^{T}\left(\begin{array}{llll}
f_{0}^{*} & f_{1}^{*} & \cdots & f_{n-1}^{*} \\
0 & & I_{n-1} & \\
& =f_{0}^{* n} X_{0}^{*}+\tilde{C}^{T}\left(\begin{array}{llll}
f_{0}^{*} & * & \cdots & \\
0 & & \left.f_{0}^{*} F^{*}\right|_{n-1} ^{a}
\end{array}\right) F^{* a} X_{F^{*}} \\
&
\end{array} X_{F^{*}},\right.
\end{aligned}
$$

using that the first row of $F^{*} F^{* a}$ equals $\left(f_{0}^{n}, 0, \cdots, 0\right)$, and abbreviating $\left.F^{*}\right|_{n-1}$ for the matrix obtained from $F^{*}$ by discarding the first row and 
column. Moreover, one can verify from (i) that $\left.F^{*}\right|_{n-1} ^{a}=\left.f_{0}^{*-1} F^{* a}\right|_{n-1}$. Discarding the first element of $X_{0}^{*}, X_{F^{*}}$, and $\tilde{C}$, we have

$$
\left.f_{0}^{* n-1} X_{0}^{*}\right|_{n-1}+\left.\left.\left.\tilde{C}^{T}\right|_{n-1} F^{*}\right|_{n-1} ^{a} X_{F^{*}}\right|_{n-1}=0,
$$

and the induction hypothesis shows that

$$
\tilde{C}^{T}=\left.\hat{C}^{T} F^{*}\right|_{n-1},
$$

for a suitable $\hat{C}$. In summary,

$$
\tilde{C}^{T}=\left(\hat{C}_{n-1}, \hat{C}_{n-2}, \cdots, \hat{C}_{0}\right)\left(\begin{array}{ll}
1 & 0 \\
0 & \left.F^{*}\right|_{n-1}
\end{array}\right),
$$

and therefore

$$
C^{* T}=\tilde{C}^{T}\left(\begin{array}{llll}
f_{0}^{*} & f_{1}^{*} & \cdots & f_{n-1}^{*} \\
0 & & I_{n-1} &
\end{array}\right)=\hat{C}^{T} F^{*} .
$$

(iii) As for the last claim, note that

$$
\begin{aligned}
0 & =\left|F^{*}\right| X_{0}^{*}+C^{* T} F^{* a} X_{F^{*}} \\
& =\left|F^{*}\right| X_{0}^{*}+\bar{C}^{* T} F^{*} F^{* a} X_{F^{*}} \\
& =\left|F^{*}\right|\left(X_{0}^{*}+\bar{C}^{T} X_{F^{*}}\right),
\end{aligned}
$$

due to $F^{*} F^{* a}=\left|F^{*}\right| I_{n}$.

Proof of Theorem 6: We assume that the vector field $X$ is of the form (10) and let $r=\max \left\{\delta X_{0}, \delta C_{i}-1\right\}$. If the degree bounds for the polynomial vector field $X_{0}$ and for the polynomials $C_{i}$ are not satisfied, then relation (11) holds. By Lemma 11 there is $\bar{C}$ such that $X_{0}^{*}=-\bar{C}^{* T} X_{F^{*}}$ and $C^{* T}=$ $\bar{C}^{*}{ }^{T} F^{*}$. We take $C^{\prime T}=C^{T}-\bar{C}^{*} F$ and $X_{0}^{\prime}=X_{0}+\bar{C}^{*} X_{F}$. Then

$$
\begin{aligned}
|F| X_{0}^{\prime}+C^{\prime T} F^{a} X_{F} & =|F| X_{0}+|F| \bar{C}^{* T} X_{F}+C^{T} F^{a} X_{F}-C^{*} T F F^{a} X_{F} \\
& =|F| X_{0}+C^{T} F^{a} X_{F} \\
& =X .
\end{aligned}
$$

Moreover, $C^{\prime *} T=C^{* T}-\bar{C}^{* T} F^{*}=0$ and $X_{0}^{*}+\bar{C}^{* T} X_{F^{*}}=0$, hence $\delta X_{0}^{\prime} \leq r$ and $\delta C_{i}^{\prime} \leq r$. Therefore, the vector field $X=|F| X_{0}^{\prime}+C^{\prime T} F^{a} X_{F}$ is of the form (10) and we have reduced the maximal degrees of $X_{0}^{\prime}$ and $C^{\prime}$ which appear in the expression. We continue this process until the bounds given in Theorem 6 are reached.

Now let us consider the case of several multiple invariant algebraic curves. We assume that the polynomial vector field $X$ admits $r$ generalized invariant algebraic curves $F^{(i)}$ of order $n_{i}$ based on curves $\left\{f_{0}^{(i)}=0\right\}$ for $i=1, \cdots, r$ 
and we define $n=n_{1}+\cdots+n_{r}$. We then define the matrix $F$ to be the block diagonal matrix with the blocks $F^{(i)}$, i.e.

(12)

$$
F=\left(\begin{array}{lll}
F^{(1)} & & 0 \\
& \ddots & \\
0 & & F^{(r)}
\end{array}\right), \quad \text { where } F^{(i)}=\left(\begin{array}{llll}
f_{0}^{(i)} & f_{1}^{(i)} & \ldots & f_{n_{i}-1}^{(i)} \\
0 & f_{0}^{(i)} & \ldots & f_{n_{i}-2}^{(i)} \\
\vdots & \ddots & \ddots & \vdots \\
0 & \ldots & 0 & f_{0}^{(i)}
\end{array}\right) .
$$

The notions defined for one multiple invariant algebraic curve can be extended as follows. Let

$$
B=\left(\begin{array}{c}
B^{(1)} \\
\vdots \\
B^{(r)}
\end{array}\right) \quad \text { where } \quad B^{(i)}=\left(\begin{array}{l}
f_{n_{i}-1}^{(i)} \\
\vdots \\
f_{0}^{(i)}
\end{array}\right)
$$

and

$$
L=\left(\begin{array}{c}
L^{(1)} \\
\vdots \\
L^{(r)}
\end{array}\right), \quad \text { with } \quad L^{(i)}=\left(\begin{array}{l}
L_{n_{i}-1}^{(i)} \\
\vdots \\
L_{0}^{(i)}
\end{array}\right)
$$

In a similar way we define the vector $B^{\prime}$ as

$$
B^{\prime}=\left(\begin{array}{c}
B^{\prime(1)} \\
\vdots \\
B^{\prime(r)}
\end{array}\right) \quad \text { where } \quad B^{\prime(i)}=\left(\begin{array}{c}
F_{n_{i}-1}^{(i)} \\
\vdots \\
F_{0}^{(i)}
\end{array}\right)
$$

and the $F_{j}^{(i)}$ are defined by $\log F^{(i)}=F_{0}^{(i)}+\epsilon F_{1}^{(i)}+\cdots$. We note that $|F|=\left|F^{(1)}\right| \cdots\left|F^{(r)}\right|=\left(f_{0}^{(1)}\right)^{n_{1}} \cdots\left(f_{0}^{(r)}\right)^{n_{r}}$ and again call $F^{a}$ the adjoint matrix of $F$. In addition, we define the vectors

$$
X_{F}=\left(\begin{array}{c}
X_{F^{(1)}} \\
\vdots \\
X_{F^{(r)}}
\end{array}\right) \quad \text { with } \quad X_{F^{(i)}}=\left(\begin{array}{c}
X_{f_{n_{i}-1}^{(i)}} \\
\vdots \\
X_{f_{0}^{(i)}}
\end{array}\right)
$$

and

$$
X_{F^{\prime}}=\left(\begin{array}{c}
X_{F^{\prime(1)}} \\
\vdots \\
X_{F^{\prime}(r)}
\end{array}\right) \quad \text { where } \quad X_{F^{\prime}(i)}=\left(\begin{array}{c}
X_{F_{n_{i}-1}^{(i)}} \\
\vdots \\
X_{F_{0}(i)}
\end{array}\right)
$$


In this case, let $I \subseteq \mathbb{C}[x, y]$ be the ideal generated by the polynomials

$$
|F|,|F| F_{k x}^{(i)},|F| F_{k y}^{(i)},|F|\left\{F_{k}^{(i)}, F_{j}^{(l)}\right\},
$$

for $k, j=1, \cdots, n_{i}-1$ and $i, l=1, \cdots, r$ where $F^{(i)}$ is defined in (12).

In what follows we use the notation

$$
C^{T}=\left(C^{(1)^{T}}, \cdots, C^{(r)^{T}}\right) \quad \text { where } C^{(i)^{T}}=\left(C_{n_{i}-1}^{(i)}, \cdots, C_{0}^{(i)}\right) \in(\mathbb{C}[x, y])^{n_{i}} .
$$

The following generalization of Theorem 5 also appears in [7].

Theorem 8. Let $F$ be the matrix (12). Then, for each $h \in I$, any polynomial vector field $X$ having the generalized invariant algebraic curves $F^{(i)}$, of order $n_{i}$ for $i=1, \cdots, r$, can be written in the form

$$
h X=|F| X_{0}+C^{T} F^{a} X_{F}=|F| X_{0}+|F| C^{T} X_{F^{\prime}},
$$

where $X_{0}$ is some polynomial vector field and $C$ is a vector of polynomials of the form (13). In particular, if $1 \in I$ then

$$
X=|F| X_{0}+C^{T} F^{a} X_{F}=|F| X_{0}+|F| C^{T} X_{F^{\prime}} .
$$

Again, under some additional conditions we obtain degree bounds for the vector fields in (15):

Theorem 9. Let $F$ be as in (12), and assume the following conditions:

(i) $I=\langle 1\rangle$.

(ii) All highest degree factors of $f_{0}^{(1)}, \cdots, f_{0}^{(r)}$ are pairwise relatively prime.

(iii) For all $i, f_{0}^{(i)}$ and $f_{1}^{(i)}$ have no common factors in the highest order terms for $i=1, \cdots, r$.

Then all polynomial vector fields $X$ having the generalized invariant algebraic curves $F^{(i)}$ of order $n_{i}$ for $i=1, \cdots, r$ can be written in the form (15) such that $X_{0}$ is a polynomial vector field with $\delta X_{0} \leq \delta X-k$ and $C$ is as in (13) with $\delta C_{j_{i}}^{(i)} \leq \delta X-k+1$ for all $i=1, \cdots, r$ and $j_{i}=0,1, \cdots, n_{i}-1$, where $k=n_{1} \cdot \delta f_{0}^{(1)}+\cdots+n_{r} \cdot \delta f_{0}^{(r)}$.

Proof: Let $s$ be the maximum of $\delta X_{0}$ and the $\delta C_{j}^{(i)}-1$, so that the highest degree occurring on the right hand side of (15) will be $s^{*}=s+k$. As above, we indicate homogeneous highest degree terms of polynomials and polynomial vector fields by an asterisk.

Suppose the degree condition is not satisfied, thus $s^{*}>\delta X$. Considering highest degree terms we find

$$
\vartheta_{0}\left|F^{*}\right| X_{0}^{*}+\sum_{i=1}^{r} \vartheta_{i} C^{*(i)^{T}} \frac{\left|F^{*}\right|}{\left|F^{*(i)}\right|}\left(F_{i}^{*}\right)^{a} X_{F_{i}^{*}}=0
$$


where we have used the block diagonal form

$$
\left(F^{*}\right)^{a}=\operatorname{diag}\left(\frac{\left|F^{*}\right|}{\left|F^{*(1)}\right|} F^{*(1)^{a}}, \cdots, \frac{\left|F^{*}\right|}{\left|F^{*(r)}\right|} F^{*(r)^{a}}\right),
$$

and set $\vartheta_{i}=1$ if the corresponding term has degree $s^{*}, \vartheta_{i}=0$ otherwise.

Assume $\vartheta_{k}=1, k \geq 1$. Then the only entry of $\frac{\left|F^{*}\right|}{\left|F^{*(k)}\right|} F^{*(k)^{a}}$ which is not divisible by $f_{0}^{*(k)}$ is in the upper right position; compare the proof of Lemma 7 and note that the highest degree terms of the $f_{0}^{(i)}$ have mutually relatively prime linear factors. As in the proof of Lemma 7 we may conclude that $f_{0}^{(k)}$ divides $C_{n_{k}-1}^{(k)}$, and this allows us to prove $C^{*(k)}=\bar{C}^{*(k) T} F^{*(k)}$ for suitable $\bar{C}^{*(k)}$, by induction. The induction starts with all $n_{i}=1$, and the relation

$$
\vartheta_{0} \prod_{j=1}^{r} f_{0}^{*(j)} X_{0}^{*}+\sum_{i=1}^{r} \vartheta_{i} C^{*(i)} \prod_{j \neq i} f_{0}^{*(j)} X_{f_{i}^{*}}=0
$$

which forces that $f_{0}^{*(k)}$ divide $C_{k}^{*}$ in case $\vartheta_{k}=1$. Then the argument runs analogously to the proof of Theorem 6 .

In order to appreciate the conditions underlying Theorems 6 and 9, we provide a geometric interpretation to the condition " $1 \in I$ ", answering a question posed in [7]. We will be satisfied to give a set of sufficient conditions, which turns out to be generic for the polynomials and curves involved. We will use the matrix $F$ in (12), and the notation introduced subsequently.

Theorem 10. Consider generalized invariant curves $F^{(i)}$ of orders $n_{i}$ based on the curves $\left\{f_{0}^{(i)}=0\right\}$, for $1 \leq i \leq r$. Assume that the following conditions are satisfied:

(1) Each $f_{0}^{(i)}$ is irreducible and each curve $\left\{f_{0}^{(i)}=0\right\}$ is nonsingular, $1 \leq$ $i \leq r$.

(2) If $n_{i}>1,1 \leq i \leq r$, then $f_{0}^{(i)}$ and $f_{1}^{(i)}$ are relatively prime and the curves $\left\{f_{0}^{(i)}=0\right\}$ and $\left\{f_{1}^{(i)}=0\right\}$ intersect transversally.

(3) If $n_{i}>2$ then $f_{0}^{(i)}, f_{1}^{(i)}$ and $f_{2}^{(i)}$ have no common zeros, $1 \leq i \leq r$.

(4) If $r>1$ then for all distinct $i, j \in\{1, \ldots, r\}, f_{0}^{(i)}$ and $f_{0}^{(j)}$ are relatively prime, and the curves $\left\{f_{0}^{(i)}=0\right\}$ and $\left\{f_{0}^{(j)}=0\right\}$ intersect transversally. Moreover, if $r>2$ then $f_{0}^{(i)}, f_{0}^{(j)}$ and $f_{0}^{(k)}$ have no common zeros for any three distinct $i, j, k$.

(5) If $r>1, i, j \in\{1, \ldots, r\}$ are distinct and $n_{i}>1$ then $f_{0}^{(i)}, f_{1}^{(i)}$ and $f_{0}^{(j)}$ have no common zeros.

Then $1 \in I$ holds. 
Proof: By the Hilbert Nullstellensatz it is sufficient to exhibit certain generators of $I$ which do not have a common zero. We will first consider the case of one generalized invariant curve $F$ based on $\left\{f_{0}=0\right\}$ of order $n$, using the notation introduced prior to Theorem 5 . Here we have to verify the assertion if (1), (2) and (3) hold. Then we will turn to the setting of several curves.

(i) From (6) we have

$$
F=f_{0} \cdot\left(I+g_{1} \varepsilon+\cdots+g_{n-1} \varepsilon^{n-1}\right),
$$

with $g_{i}=f_{i} / f_{0}$, and therefore

$$
\begin{aligned}
\log F & =F_{0} \cdot I+F_{1} \cdot \varepsilon+\cdots+F_{n-1} \varepsilon^{n-1} \\
& =\log f_{0} \cdot I+N-\frac{1}{2} N^{2}+\frac{1}{3} N^{3} \cdots+\frac{(-1)^{n}}{n-1} N^{n-1}+\cdots
\end{aligned}
$$

with $N:=\sum_{i=1}^{n-1} g_{i} \varepsilon^{i}$. Since we can write $N^{k}$ as

$$
N^{k}=\sum_{\ell}\left(\sum_{i_{1}+\cdots+i_{k}=\ell} g_{i_{1}} \cdots g_{i_{k}}\right) \varepsilon^{\ell},
$$

with summation over tuples $\left(i_{1}, \cdots, i_{k}\right) \in \mathbb{Z}_{+}^{k}$, rearranging yields

$$
F_{\ell}=\sum_{k}^{n-1} \frac{(-1)^{k+1}}{k}\left(\sum_{i_{1}+\cdots+i_{k}=\ell} g_{i_{1}} \cdots g_{i_{k}}\right) .
$$

We note

$$
\left(g_{i_{1}} \cdots g_{i_{k}}\right)_{x}=\frac{1}{f_{0}^{k+1}} \sum_{m=1}^{k}\left(f_{i_{1}} \cdots \widehat{f_{i_{m}}} \cdots f_{i_{k}}\right)\left(f_{i_{m} x} f_{0}-f_{0 x} f_{i_{m}}\right),
$$

where "?" symbolizes deletion, and similarly for the partial derivatives with respect to $y$. Moreover one verifies

$$
\text { (19) }\left\{\log f_{0}, g_{i}\right\}=\frac{1}{f_{0}^{2}}\left\{f_{0}, f_{i}\right\}
$$

for all $i, j \in\{1, \ldots, n\}$.

(ii) Now assume that $z$ is a zero of $I$. Then $f_{0}(z)^{n}=0$, hence $f_{0}(z)=0$. From (16) we see that

$$
F_{n-1}=\frac{(-1)^{n}}{n-1} g_{1}^{n-1}+\frac{1}{f_{0}^{n-2}} \cdot q,
$$


for some polynomial $q$, and hence

$$
\begin{aligned}
& 0=\left(|F| \cdot F_{n-1, x}\right)(z)=(-1)^{n+1} f_{1}(z)^{n-1} f_{0 x}(z), \\
& 0=\left(|F| \cdot F_{n-1, y}\right)(z)=(-1)^{n+1} f_{1}(z)^{n-1} f_{0 y}(z),
\end{aligned}
$$

by (17). Since the curve $\left\{f_{0}=0\right\}$ is non singular, we find that $f_{1}(z)=0$.

(iii) Now consider $|F| \cdot\left\{F_{n-1}, F_{n-2}\right\}$, which is a polynomial by [7], Lemma 5.9. Since $F_{\ell}$ has the representation (16), we have to deal with Poisson brackets of type

$$
p=\left\{g_{i_{1}} \cdots g_{i_{k}}, g_{j_{1}} \cdots g_{j_{m}}\right\} .
$$

By the Leibniz rule and (18), expanding this term will produce a rational function with denominator $f_{0}^{k+m+1}$. We are only interested in the case $k+m \geq n-1$, since $|F| \cdot p$ is a polynomial multiple of $f_{0}$ in case $k+m<n-1$, and such terms will vanish upon substituting a zero $z$ of $I$.

(iv) In case $n=2$ we have

$$
|F| \cdot\left\{F_{1}, F_{0}\right\}=\left\{f_{1}, f_{0}\right\}
$$

by (16) and (19). Since any zero of $I$ must be a common zero of $f_{0}, f_{1}$ and $\left\{f_{1}, f_{0}\right\}$ and this contradicts the transversality condition, we conclude that $I$ admits no zero.

Now assume that $n>2$ is even. Then the representation of $F_{n-1}$ contains the term $g_{1} g_{2}^{(n-2) / 2}$ (with $n / 2$ factors) and the representation of $F_{n-2}$ contains the term $g_{2}^{(n-2) / 2}$ (with $(n-2) / 2$ factors), both with nonzero coefficients. By (18) we have

$$
\begin{aligned}
|F| & \cdot\left\{g_{1} g_{2}^{(n-2) / 2}, g_{2}^{(n-2) / 2}\right\}= \\
& =|F| \cdot g_{2}^{(n-2) / 2} \cdot \frac{n-2}{2} g_{2}^{(n-4) / 2} \cdot\left\{g_{1}, g_{2}\right\} \\
& =\frac{n-2}{2} f_{2}^{n-3}\left(f_{0}\left\{f_{1}, f_{2}\right\}-f_{2}\left\{f_{1}, f_{0}\right\}-f_{1}\left\{f_{0}, f_{2}\right\}\right) .
\end{aligned}
$$

Substituting a zero $z$ of $I$ yields $f_{2}(z)\left\{f_{1}, f_{0}\right\}(z) \neq 0$, since $z$ is a transversal intersection point of $\left\{f_{0}=0\right\}$ and $\left\{f_{1}=0\right\}$, and $f_{2}$ does not vanish at $z$. We will show next: All other relevant Poisson brackets $p$ as in (iii) which involve a total of $n-1$ or more factors will contain a term $g_{1}^{d}$ with some $d>1$ in one of the entries. By the Leibniz rule we then have

$$
|F| \cdot p=g_{1} \cdot p^{*}=f_{1} \cdot q,
$$

with a suitable polynomial $p^{*}$ and a suitable rational function $q$. Substitution of a zero $z$ of $I$ will thus yield 0 for each such bracket, and in total we find

$$
\left(|F| \cdot\left\{F_{n-1}, F_{n-2}\right\}\right)(z) \neq 0,
$$


which is in contradiction with the assumption that $I$ admits the zero $z$. We will be finished when Claim 1 and Claim 2 below are proven.

Claim 1. In the representation (16) for $F_{n-1}$, every term with more than $n / 2$ factors, as well as every term with $n / 2$ factors that is different from $g_{1} g_{2}^{(n-2) / 2}$, contains a factor $g_{1}^{d}$ with $d>1$.

To prove this, assume $i_{1}+\cdots+i_{k}=n-1$. If all $i_{s} \geq 2$ then $n-1 \geq 2 k$ and $n-2 \geq 2 k$ because $n$ is even. So, $(n-2) / 2 \geq k$. Thus, for $k \geq n / 2$ there is at least one factor $g_{1}$. Furthermore, assume that, for instance, $i_{1}=1$ and all other $i_{s} \geq 2$. Then $n-2 \geq 2(k-1)$, so $n / 2 \geq k$. Thus, for $k>n / 2$ we must have a factor $g_{1}^{d}$ with some $d>1$. If $k=n / 2$ and there is a simple factor $g_{1}$ (thus all $i_{s} \geq 2$ for $s>1$ ), we find

$$
n-2=i_{2}+\cdots+i_{n / 2} \geq((n-2) / 2) \cdot 2=n-2,
$$

which is possible only for $i_{2}=\ldots=i_{n / 2}=2$. Hence, Claim 1 is proved. Claim 2. In the representation (16) for $F_{n-2}$, every term with more than $(n-2) / 2$ factors, as well as every term with $(n-2) / 2$ factors that is different from $g_{2}^{(n-2) / 2}$, contains a factor $g_{1}^{d}$ with $d>1$.

The proof is an obvious variant of the proof of Claim 1.

(v) If $n>1$ is odd, then repeat (iv) with the terms $g_{2}^{(n-1) / 2}$ in $F_{n-1}$ and $g_{1} \cdot g_{2}^{(n-3) / 2}$ in $F_{n-2}$, with straightforward modifications.

Thus the case of one multiple curve is settled: If (1), (2) and (3) hold then $1 \in I$.

(vi) Now we turn to several multiple curves. Clearly, a zero $z$ of $I$ is also a zero of $f_{0}^{(1)} \cdots f_{0}^{(r)}$, and substituting $z$ into

$$
|F| \cdot F_{n_{i}-1, x}^{(i)} \text { and }|F| \cdot F_{n_{i}-1, y}^{(i)}
$$

shows that a zero $z$ of $I$ with $f_{0}^{(i)}(z)=0$ satisfies $f_{0}^{(j)}(z)=0$ for some $j \neq i$ or $f_{1}^{(i)}(z)=0$; compare the argument in part (ii). In the latter case we have $\prod_{j \neq i} f_{0}^{(j)}(z) \neq 0$, and just as in steps (iii) through (v) we obtain a contradiction.

In the first case, assume $f_{0}^{(i)}(z)=f_{0}^{(j)}(z)=0$ and consider

$$
|F| \cdot\left\{\left(\frac{f_{1}^{(i)}}{f_{0}^{(i)}}\right)^{n_{i}-1},\left(\frac{f_{1}^{(j)}}{f_{0}^{(j)}}\right)^{n_{j}-1}\right\}
$$

if $n_{i}>1$ and $n_{j}>1$. This term is equal to

$$
\prod_{k \neq i, j}\left|F^{(k)}\right| \cdot\left(\left(n_{i}-1\right)\left(n_{j}-1\right) \cdot f_{1}^{(i)} f_{1}^{n_{i}-2} f^{(j)^{n_{j}-2}} \cdot\left\{f_{0}^{(i)}, f_{0}^{(j)}\right\}+f_{0}^{(i)} \cdot(\cdots)+f_{0}^{(j)} \cdot(\cdots)\right) \text {. }
$$


Upon substituting $z$, we obtain a nonzero first factor, and a nonzero first term inside the bracket, while the second and third terms vanish. Again the assumption that $I$ admits a zero leads to a contradiction.

In case $n_{i}=1$ or $n_{j}=1$, replace the corresponding term in the Poisson bracket by $\log f_{0}^{(i)}$ or $\log f_{1}^{(i)}$, respectively, and an obvious modification of the above argument applies.

\section{DARBOUX INTEGRALS AND INTEGRATING FACTORS}

We now turn to a more special inverse problem: describe the vector space of polynomial vector fields which admit a given Darboux integrating factor. Using the notation introduced in the previous section, a Darboux inverse integrating factor formed by $r$ multiple curves based on $f^{(1)} \cdots f^{(r)}$ can be written in the form

(20)

$$
\begin{aligned}
V & =\exp \left(\lambda \cdot B^{\prime}\right)=\exp \left(\lambda^{(1)} \cdot B^{\prime(1)}\right) \cdots \exp \left(\lambda^{(r)} \cdot B^{\prime(r)}\right) \\
& =\left(f_{0}^{(1)}\right)^{\lambda_{0}^{(1)}} \prod_{j=1}^{n_{1}-1} \exp \left(\lambda_{j}^{(1)} F_{j}^{(1)}\right) \cdots\left(f_{0}^{(r)}\right)^{\lambda_{0}^{(r)}} \prod_{j=1}^{n_{r}-1} \exp \left(\lambda_{j}^{(r)} F_{j}^{(r)}\right),
\end{aligned}
$$

with $n_{1} \geq 1, \cdots, n_{r} \geq 1$, where a product over an empty index set equals 1. We have introduced the abbreviations $\lambda=\left(\lambda^{(1)}, \cdots, \lambda^{(r)}\right)$ where $\lambda^{(i)}=$ $\left(\lambda_{n_{i}-1}^{(i)}, \cdots, \lambda_{0}^{(i)}\right) \in \mathbb{C}^{n_{i}}$, and furthermore introduce $\alpha=\left(\alpha^{(1)}, \cdots, \alpha^{(r)}\right)$ where $\alpha^{(i)}=\left(0, \cdots, 0, n_{i}\right) \in \mathbb{C}^{n_{i}}, e_{k}=\left(e_{k}^{(1)}, \cdots, e_{k}^{(r)}\right)$ where $e_{k}^{(i)}=\left(0, \cdots, 0, \delta_{k j}\right) \in \mathbb{C}^{n_{j}}$, for $1 \leq k \leq r$. We always require $\lambda_{n_{k}-1}^{(k)} \neq 0$ if $n_{k}>1$. Clearly, this involves no loss of generality.

As a first step we exhibit some polynomial vector fields that admit the inverse integrating factor $V$ from (20). For a given polynomial $g$, denote by

$$
Z_{g}^{\lambda}=X_{g \exp \left[(\alpha-\lambda) \cdot B^{\prime}\right]}
$$

the Hamiltonian vector field of the function $g \exp \left[(\alpha-\lambda) \cdot B^{\prime}\right]$. We collect some properties of such vector fields:

Lemma 11. (a) One has

$$
Z_{g}^{\lambda}=\exp \left(-\lambda \cdot B^{\prime}\right)\left(|F| X_{g}+g|F|(\alpha-\lambda) \cdot X_{F^{\prime}}\right),
$$

and therefore $\exp \left(\lambda \cdot B^{\prime}\right) Z_{g}^{\lambda}$ is a polynomial vector field which admits the Darboux inverse integrating factor $V$ and the Darboux first integral $g \exp \left((\alpha-\lambda) \cdot B^{\prime}\right)$.

(b) Given integers $d_{1}, \cdots, d_{r} \geq 0$, the identity

$$
Z_{g}^{\lambda-\sum d_{k} e_{k}}=Z_{g^{*}}^{\lambda}
$$


holds for $g^{*}=g \cdot\left(f_{0}^{(1)}\right)^{d_{1}} \cdots\left(f_{0}^{(r)}\right)^{d_{r}}$

Proof: (a) Using

$$
\exp \left[(\alpha-\lambda) \cdot B^{\prime}\right]=\exp \left(\alpha \cdot B^{\prime}\right) \exp \left(-\lambda \cdot B^{\prime}\right)=|F| \exp \left(-\lambda \cdot B^{\prime}\right),
$$

we find

$$
\begin{aligned}
Z_{g}^{\lambda} & =\exp \left[(\alpha-\lambda) \cdot B^{\prime}\right] X_{g}+g \exp \left[(\alpha-\lambda) \cdot B^{\prime}\right] X_{(\alpha-\lambda) \cdot B^{\prime}} \\
& =\exp \left(-\lambda \cdot B^{\prime}\right)\left(|F| X_{g}+g|F| X_{(\alpha-\lambda) \cdot B^{\prime}}\right) \\
& =\exp \left(-\lambda \cdot B^{\prime}\right)\left(|F| X_{g}+g|F|(\alpha-\lambda) \cdot X_{F^{\prime}}\right)
\end{aligned}
$$

by various definitions. As noted earlier, all $|F| X_{F_{j}^{(i)}}$ are polynomial vector fields, and the first assertion is proved. The last two assertions are obvious. (b) Since $Z_{g}^{\lambda-e_{k}}$ is the Hamiltonian vector field of

$$
g \exp \left[\left(\alpha+e_{k}-\lambda\right) \cdot B^{\prime}\right]=g f_{0}^{(k)}|F| \exp \left(-\lambda \cdot B^{\prime}\right)=\left(g f_{0}^{(k)}\right) \exp \left((\alpha-\lambda) \cdot B^{\prime}\right),
$$

we have

$$
Z_{g}^{\lambda-e_{k}}=Z_{g f_{0}^{(k)}}^{\lambda}
$$

The assertion now follows by simple induction.

Theorem 12. Assume that conditions (1) to (5) of Theorem 10 hold, and $X$ is a polynomial vector field which admits the inverse integrating factor $V$ given by $(20)$.

(a) If some $n_{k}>1$, or $n_{1}=\cdots=n_{r}=1$ but some $\lambda_{0}^{(k)}$ is not a positive integer, and the additional conditions of Theorem 9 are satisfied then $X=\exp \left(\lambda \cdot B^{\prime}\right) Z_{g}^{\lambda}$ for some polynomial $g$, and $X$ admits the Darboux first integral $g \exp \left((\alpha-\lambda) \cdot B^{\prime}\right)$.

(b) If $n_{1}=\cdots=n_{r}=1$ and all $\lambda_{0}^{(j)}$ are positive integers then

$$
X=|F| Z_{h}^{\lambda}+|F| X_{\tilde{G}}-|F| \sum_{i=1}^{r} \beta_{i} \frac{X_{f_{0}^{(i)}}}{f_{0}^{(i)}},
$$

for certain polynomials $h$ and $\tilde{G}$. In this case, $X$ admits a Darboux first integral

$$
\exp (G) \prod_{i=1}^{r}\left(f_{0}^{(i)}\right)^{\beta_{i}},
$$

with some rational function $G$. The denominator of $G$ is a product of powers of the $f_{0}^{(i)}$. 
Proof: According to Theorem 8 we can write $X$ in the form

$$
\begin{aligned}
X & =|F| X_{0}+|F| C^{T} X_{F^{\prime}} \\
& =|F| X_{0}+|F| \sum_{i=1}^{r} \sum_{j=0}^{n_{i}-1} C_{j}^{(i)} X_{F_{j}^{(i)}}
\end{aligned}
$$

with degree bounds on the $C_{j}^{(i)}$ if the additional hypotheses of Theorem 9 hold.

(i) We first establish a reduction principle.

If some $n_{l}>1$ (thus $\lambda_{n_{l}-1}^{(l)} \neq 0$ ), define $g=\frac{C_{n_{k-1}}^{(k)}}{\lambda_{n_{k}-1}^{(k)}}$, to be such that $n_{k}>1$ and $C_{n_{k}-1}^{(k)}$ has the smallest degree among the $C_{n_{l}-1}^{(l)}$ with $n_{l}>1$.

If $n_{1}=\cdots=n_{r}=1$, but some $\lambda_{0}^{(k)} \neq 1$, define $g=\frac{C_{0}^{(k)}}{\lambda_{0}^{(k)}-1}$.

Then

$$
\begin{aligned}
\exp \left(\lambda \cdot B^{\prime}\right) Z_{g}^{\lambda} & =|F| X_{g}+g|F| \sum_{i, j}\left(\alpha_{j}^{(i)}-\lambda_{j}^{(i)}\right) X_{F_{j}^{(i)}} \\
& =|F| X_{g}-\left(\alpha_{n_{k}-1}^{(k)}+\lambda_{n_{k}-1}^{(k)}\right) g|F| X_{F_{n_{k}-1}^{(k)}} \\
& +g|F| \sum_{(i, j) \neq\left(k, n_{k}-1\right)}\left(\alpha_{j}^{(i)}-\lambda_{j}^{(i)}\right) X_{F_{j}^{(i)}} .
\end{aligned}
$$

Now $\alpha_{n_{k}-1}^{(k)}-\lambda_{n_{k}-1}^{(k)}=-\lambda_{n_{k}-1}^{(k)}$ if $n_{k}>1$ and thus $\left(\alpha_{n_{k}-1}^{(k)}-\lambda_{n_{k}-1}^{(k)}\right) g=$ $-C_{n_{k}-1}^{(k)}$.

In case $n_{k}=1, \lambda_{0}^{(k)} \neq 1$, we have $\alpha_{n_{k}-1}^{(k)}-\lambda_{n_{k}-1}^{(k)}=1-\lambda_{0}^{(k)}$, and again $\left(\alpha_{n_{k}-1}^{(k)}-\lambda_{n_{k}-1}^{(k)}\right) g=-C_{n_{k}-1}^{(k)}$. Thus in both cases we arrive at a polynomial vector field

$$
X+\exp \left(\lambda \cdot B^{\prime}\right) Z_{g}^{\lambda}=|F|\left(X_{0}+X_{g}\right)+|F| \sum_{(i, j) \neq\left(k, n_{k}-1\right)} \tilde{C}_{j}^{(i)} X_{F_{j}^{(i)}},
$$

with $\tilde{C}_{j}^{(i)}=C_{j}^{(i)}+g\left(\alpha_{j}^{(i)}-\lambda_{j}^{(i)}\right)$.

If the hypotheses of Theorem 9 hold then, due to the degree bounds on the $C_{j}^{(i)}$ and the choice of $C_{n_{k}-1}^{(k)}$, the degree of $X+\exp \left(\lambda \cdot B^{\prime}\right) Z_{g}^{\lambda}$ is not greater than the degree of $X$.

Furthermore, $f_{0}^{(k)}$ divides each term on the right-hand side, since the denominator of $X_{F_{j}^{(i)}}$ does not contain $f_{0}^{(k)}$ for $i \neq k$, and the denominator of $X_{F_{j}^{(k)}}$ contains $f_{0}^{(k)}$ to a power less than $n_{k}$ unless $j=n_{k}$; see Remark 
5.10 of [7]. Therefore, we obtain

$$
X+\exp \left(\lambda \cdot B^{\prime}\right) Z_{g}^{\lambda}=f_{0}^{(k)} \hat{X}
$$

and $\hat{X}$ is a polynomial vector field which obviously admits the inverse integrating factor $\hat{V} \cdot\left(f_{0}^{(k)}\right)^{-1}=\exp \left(\left(\lambda-e_{k}\right) \cdot B^{\prime}\right)$. Moreover, $\delta \hat{X}<\delta X$ if the hypotheses of Theorem 9 hold.

(ii) Assume that some $n_{j}>1$ or $n_{1}=\cdots=n_{r}=1$ but some $\lambda_{0}^{(k)}$ is not a positive integer. Then we can repeat the reduction procedure an arbitrary number of times. Since the hypotheses of Theorem 9 continue to hold for the vector fields remaining after the reduction step, the strict descent of degrees ensures that one will eventually arrive at the zero vector field. All vector fields added in the process are of type $\exp \left(\lambda \cdot B^{\prime}\right) Z_{h}^{\lambda}$ for some polynomial $h$, by Lemma 11 . Thus, part (a) is proven.

(iii) Now assume that $n_{1}=\cdots=n_{r}=1$ and all $\lambda_{0}^{(k)}=d_{k}$ are positive integers. Here we can reduce until all $\lambda_{0}^{(k)}=1$, and then have, abbreviating $f_{i}=f_{0}^{(i)}$

$$
X=|F| Z_{h}^{\lambda}+\prod_{i=1}^{r} f_{i}^{d_{i}-1} \tilde{X}
$$

where $h$ is some polynomial and $\tilde{X}$ a polynomial vector field with integrating factor $\left(f_{1} \cdots f_{r}\right)^{-1}$.

Since $1 \in I$, the vector field $\tilde{X}$ can be written in the form (14), i.e.

$$
\tilde{X}=f_{1} \cdots f_{r} X_{0}+f_{1} \cdots f_{r} \sum_{i=1}^{r} C_{i} \frac{X_{f_{i}}}{f_{i}},
$$

and $\tilde{X}$ has inverse integrating factor $V=f_{1} \cdots f_{r}$, whence $\operatorname{div}\left(X /\left(f_{1} \cdots f_{r}\right)\right)=0$, or

$$
0=\operatorname{div}\left(X_{0}\right)+\sum_{i=1}^{r} \frac{1}{f_{i}}\left\{f_{i}, C_{i}\right\}
$$

Since the $f_{i}$ are relatively prime polynomials, each $f_{i}$ must divide $\left\{f_{i}, C_{i}\right\}$. Hence, the Hamiltonian vector field $X_{C}=\left(-C_{i y}, C_{i x}\right)$ has $f_{i}=0$ as invariant algebraic curve. Therefore, $\left\{f_{i}=0\right\} \subseteq\left\{C_{i}-\beta_{i}=0\right\}$ for some constant $\beta_{i} \in \mathbb{C}$, for all $i=1, \cdots, r$. From the Hilbert Nullstellensatz, and because $f_{i}$ is irreducible we get $C_{i}-\beta_{i}=M_{i} f_{i}$ for some $M_{i} \in \mathbb{C}[x, y], i=1, \cdots, r$. Using

$$
\operatorname{div}\left(\sum_{i=1}^{r} M_{i} X_{f_{i}}\right)=\sum_{i=1}^{r}\left\{f_{i}, M_{i}\right\}=\sum_{i=1}^{r} \frac{1}{f_{i}}\left\{f_{i}, C_{i}\right\}
$$


the relation (26) becomes

$$
\operatorname{div}\left(X_{0}+\sum_{i=1}^{r} M_{i} X_{f_{i}}\right)=0
$$

and we conclude

$$
X_{0}+\sum_{i=1}^{r} M_{i} X_{f_{i}}=X_{\tilde{G}},
$$

for some polynomial $\tilde{G}$. Substituting $X_{0}$ into the expression $(25)$ we get

$$
\tilde{X}=f_{1} \cdots f_{r} X_{\tilde{G}}-f_{1} \cdots f_{r} \sum_{i=1}^{r} \beta_{i} \frac{X_{f_{i}}}{f_{i}},
$$

and thus

$$
X=|F| Z_{h}^{\lambda}+|F| X_{\tilde{G}}-|F| \sum_{i=1}^{r} \beta_{i} \frac{X_{f_{i}}}{f_{i}} .
$$

Therefore, $X$ admits the first integral

$$
\begin{aligned}
H & =h \exp \left((\alpha-\lambda) \cdot B^{\prime}\right)+\tilde{G}-\sum \beta_{i} \log f_{i} \\
& =h f_{1}^{1-d_{1}} \cdots f_{r}^{1-d_{r}}+\tilde{G}-\sum \beta_{i} \log f_{i} \\
& =: \quad G-\sum \beta_{i} \log f_{i}
\end{aligned}
$$

with a rational function $G$ whose denominator is a product of powers of the $f_{i}$, and hence the Darboux first integral $\exp (H)=\exp (G) f_{1}^{-\beta_{1}} \cdots f_{r}^{-\beta_{r}}$.

In Case (b) of the Theorem, a more general result can be stated and proven.

Proposition 13. If the vector field given by (14) admits an inverse integrating factor of the form $V=f_{1} \cdots f_{r}$ with irreducible and pairwise relatively prime $f_{i}$ then it has a Darboux first integral of the form $\exp (G) \prod_{i=1}^{r} f_{i}^{\alpha_{i}}$, where $G$ is a rational function and $\alpha_{i} \in \mathbb{C}$.

Proof: The vector field admits the invariant algebraic curves $\left\{f_{i}=0\right\}$ for all $i$. Since the ideal $I$ contains the elements

$$
\left(\prod_{i \neq k} f_{i}\right) \cdot f_{k x}, \quad\left(\prod_{i \neq k} f_{i}\right) \cdot f_{k y},
$$

and each $f_{k}$ is irreducible, there exists a polynomial $h \in I$ which is not a multiple of any $f_{k}$. For this $h$, Theorem 8 shows the existence of a polynomial vector field $X_{0}$ and polynomials $C_{i}$ such that

$$
h X=V X_{0}+V \sum_{i=1}^{r} C_{i} \frac{X_{f_{i}}}{f_{i}},
$$


or

$$
X=V X_{0}^{\prime}+V \sum_{i=1}^{r} C_{i}^{\prime} \frac{X_{f_{i}}}{f_{i}}
$$

with $X_{0}^{\prime}=X_{0} / h$ and $C_{i}^{\prime}=C_{i} / h$. In the following we use familiar properties of the ring $R:=\mathbb{C}[x, y]\left[\frac{1}{h}\right]$, which is the coordinate ring of an affine variety, viz. the principal open subset defined by $h \neq 0$; see [13], Ch. 1, §4. In particular the Hilbert Nullstellensatz continues to hold over this ring, and the ideal generated by $f_{i}$ remains prime in $R$ by choice of $h$. Since the vector field $X$ has the inverse integrating factor $V$ we get $\operatorname{div}(X / V)=0$, or equivalently,

$$
0=\operatorname{div}\left(X_{0}^{\prime}\right)+\sum_{i=1}^{r} \frac{1}{f_{i}}\left\{f_{i}, C_{i}^{\prime}\right\} .
$$

Repeating the argument in part (iii) of the proof of Theorem 12, we obtain $C_{i}^{\prime}=\theta_{i}+M_{i}^{\prime} f_{i}$ with suitable $\theta_{i} \in \mathbb{C}$ and $M_{i}^{\prime} \in R$. Because of

$$
\operatorname{div}\left(\sum_{i=1}^{r} M_{i}^{\prime} X_{f_{i}}\right)=\sum_{i=1}^{r}\left\{f_{i}, M_{i}^{\prime}\right\}=\sum_{i=1}^{r} \frac{1}{f_{i}}\left\{f_{i}, C_{i}^{\prime}\right\},
$$

relation (28) becomes

$$
\operatorname{div}\left(X_{0}^{\prime}+\sum_{i=1}^{r} M_{i}^{\prime} X_{f_{i}}\right)=0
$$

and $\hat{X}=X_{0}^{\prime}+\sum_{i=1}^{r} M_{i}^{\prime} X_{f_{i}}$ is a rational vector field. By a well-known result, see e.g. [3], we have that $\hat{X}=X_{\log F}$ where $F$ is a Darboux function of the form $F=\left(\prod_{i=1}^{r} f_{i}^{\beta_{i}}\right) \exp G$ with $\beta_{i} \in \mathbb{C}$, and $G$ is a rational function. Thus

$$
\hat{X}=\sum_{i=1}^{r} \beta_{i} \frac{X_{f_{i}}}{f_{i}}+X_{G}
$$

and moreover

$$
X_{0}^{\prime}+\sum_{i=1}^{r} M_{i}^{\prime} X_{f_{i}}=X_{G}+\sum_{i=1}^{r} \beta_{i} \frac{X_{f_{i}}}{f_{i}} .
$$

Substituting $X_{0}^{\prime}$ into relation (27) we obtain

$$
X=V X_{G}-V \sum_{i=1}^{r} \theta_{i} \frac{X_{f_{i}}}{f_{i}}+V \sum_{i=1}^{r} \beta_{i} \frac{X_{f_{i}}}{f_{i}} .
$$

This vector field $X$ has the Darboux first integral $\exp (G) \prod_{i=1}^{r} f_{i}^{\theta_{i}-\beta_{i}}$. 


\section{EXAmPles AND CONTEREXAMPLES}

In this section we use Lemma 2 for an approach to the construction of vector fields with multiple invariant curves. We are particularly interested in cases when the genericity condition " $1 \in I$ " from Section 3 is not satisfied.

We observe that the form of the relations (5) suggests a step by step approach: First, find all polynomial vector fields that satisfy $X\left(f_{0}\right)=L_{0} f_{0}$ for some polynomial $L_{0}$, then rewrite the second condition as

$$
X\left(f_{1}\right)-L_{0} f_{1} \in\left\langle f_{0}\right\rangle,
$$

which will impose further restrictions on $X$ and $L_{0}$, and proceed. The intermediate condition

$$
X\left(f_{1}\right)=L_{2} f_{1}+L_{1} f_{0} \in\left\langle f_{0}, f_{1}\right\rangle,
$$

yields some properties of $X$ that facilitate the subsequent work. Here Lemma 2 is useful.

In our first example, $f_{0}$ is reducible and the components of $\left\{f_{0}=0\right\}$ intersect.

Proposition 14. For $f_{0}=x y, f_{1}=1+x+y$ the following hold:

(a) A polynomial vector field $X$ satisfies

$$
\begin{aligned}
& X\left(f_{0}\right)=L_{0} f_{0}, \\
& X\left(f_{1}\right)=L_{0} f_{1}+L_{1} f_{0},
\end{aligned}
$$

for suitable polynomials $L_{0}$ and $L_{1}$ if and only if

$$
X=\alpha\left(\begin{array}{c}
x^{2} y \\
0
\end{array}\right)+B\left(f_{1} X_{f_{0}}-f_{0} X_{f_{1}}\right)+C f_{0} X_{f_{0}}+f_{0}^{2} X_{0},
$$

with $\alpha \in \mathbb{C}, B$ and $C$ arbitrary polynomials and $X_{0}$ an arbitrary polynomial vector field. For $\alpha=0$ this includes only the terms given in Theorem 5, but for $\alpha \neq 0$ the vector field has no such representation.

(b) If $X$ admits an integrating factor of type

$$
f_{0}^{-\lambda_{0}}\left(\exp \left(\frac{f_{1}}{f_{0}}\right)\right)^{-\lambda_{1}},
$$

then $\lambda_{1}=0$ or $\alpha=0$. In both cases there is a Darboux first integral.

Proof: (i) Since $f_{0}=x y$, and $\{x=0\}$ and $\{y=0\}$ intersect transversally we have $X\left(f_{0}\right) \in\left\langle f_{0}\right\rangle$ if and only if

$$
X=A_{1} y \frac{\partial}{\partial y}-A_{2} x \frac{\partial}{\partial x}+x y \tilde{X}_{0}
$$


see Lemma 7(a) of [6]. Moreover, $\left\{f_{0}=0\right\} \cap\left\{f_{1}=0\right\}=\{(-1,0),(0,-1)\}$. Stationarity of these points, as required by Lemma 2 , is equivalent to $A_{1}(0,-1)=A_{2}(-1,0)=0$, so

$$
A_{1}=(y+1) \tilde{A_{1}}, \quad A_{2}=(x+1) \tilde{A_{2}},
$$

which implies

$$
X=y(y+1) \tilde{A}_{1} \frac{\partial}{\partial y}-x(x+1) \tilde{A}_{2} \frac{\partial}{\partial x}+x y \tilde{X}_{0} .
$$

The cofactor of $f_{0}$ is equal to

$$
L_{0}=(y+1) \tilde{A}_{1}-(x+1) \tilde{A}_{2}+y \tilde{P}_{0}+x \tilde{Q}_{0},
$$

with $\tilde{X}_{0}=\left(\tilde{P}_{0}, \tilde{Q}_{0}\right)$.

(ii) For (a) the necessary and sufficient condition is

$$
X\left(f_{1}\right)-L_{0} f_{1} \in\left\langle f_{0}\right\rangle,
$$

or equivalently

$-y(1+x+y) \tilde{P}_{0}-x(1+x+y) \tilde{Q}_{0}-(1+x)(1+y) \tilde{A}_{1}+(1+x)(1+y) \tilde{A}_{2} \in\langle x y\rangle$, as a brief computation shows. This is equivalent to

$-y(1+x+y) \tilde{P}_{0}-x(1+x+y) \tilde{Q}_{0}-(1+x+y) \tilde{A}_{1}+(1+x+y) \tilde{A}_{2} \in\langle x y\rangle$, and we have the necessary and sufficient condition

$$
\tilde{A}_{2}=\tilde{A}_{1}+y \tilde{P}_{0}+x \tilde{Q}_{0}+x y R,
$$

for some polynomial $R$. Substituting this in $X=(P, Q)$ we obtain

$$
\begin{aligned}
\left(\begin{array}{c}
P \\
Q
\end{array}\right)= & \tilde{A}_{1}\left(\begin{array}{r}
-x(x+1) \\
y(y+1)
\end{array}\right)-\tilde{P}_{0}\left(\begin{array}{c}
x^{2} y \\
0
\end{array}\right)+ \\
& +\tilde{Q}_{0}\left(\begin{array}{c}
-x^{3}+x^{2} \\
x y
\end{array}\right)+\left(-R+\tilde{P}_{0}\right)\left(\begin{array}{c}
x^{3} y \\
0
\end{array}\right)= \\
= & A_{1}\left(\begin{array}{r}
-x(x+1) \\
y(y+1)
\end{array}\right)+\hat{P}_{0}\left(\begin{array}{c}
x^{2} y \\
0
\end{array}\right)+ \\
& +\hat{Q}_{0}\left(\begin{array}{c}
0 \\
x y^{2}
\end{array}\right)+\hat{R}\left(\begin{array}{c}
x^{3} y \\
0
\end{array}\right),
\end{aligned}
$$

with $\tilde{A}_{1}=A_{1}-x \tilde{Q_{0}}$ and obvious renaming.

(iii) Vector fields of the form $X^{*}=\left(P^{*}, Q^{*}\right)$ with

$$
\left(\begin{array}{c}
P^{*} \\
Q^{*}
\end{array}\right)=B\left(\begin{array}{c}
-x(x+1) \\
y(y+1)
\end{array}\right)+C\left(\begin{array}{c}
-x^{2} y \\
x y^{2}
\end{array}\right)+x^{2} y^{2}\left(\begin{array}{c}
P_{0}^{*} \\
Q_{0}^{*}
\end{array}\right),
$$


clearly admit $f_{0}+\varepsilon f_{1}$ as a generalized invariant algebraic curve, being in the form of Theorem 5 .

We claim that the vector field $\left(x^{2} y, 0\right)$ cannot be written in the form (30). To prove this claim, assume that $\left(x^{2} y, 0\right)$ is written in such a form, thus we have

$$
\begin{aligned}
& x^{2} y=-B x-B x^{2}-C x^{2} y+x^{2} y^{2} P_{0}^{*}, \\
& 0=B y+B y^{2}+C x y^{2}+x^{2} y^{2} Q_{0}^{*},
\end{aligned}
$$

or equivalently

$$
\begin{aligned}
& x y=-B-B x-C x y+x y^{2} P_{0}^{*} \\
& 0=B+B y+C x y+x^{2} y Q_{0}^{*} .
\end{aligned}
$$

From the first equation of (31) we find $B=\hat{B} x$, and so we can rewrite the second equation as

$$
0=\hat{B}+\hat{B} y+C y+x y Q_{0}^{*},
$$

which implies $\hat{B}=\hat{\hat{B}} y$ as well as $\hat{\hat{B}}=-C(\bmod \langle x, y\rangle)$, thus we have $B=-C x y+$ higher order terms. Substituting this in the first equation of (31) yields a contradiction, and the claim is proved.

Since

$$
\left(\begin{array}{c}
x^{3} y \\
0
\end{array}\right)=x\left(\begin{array}{c}
x^{2} y \\
-x y^{2}
\end{array}\right)+x^{2} y^{2}\left(\begin{array}{l}
0 \\
1
\end{array}\right),
$$

and

and

$$
\left(\begin{array}{c}
0 \\
x y^{3}
\end{array}\right)=-y\left(\begin{array}{c}
x^{2} y \\
-x y^{2}
\end{array}\right)+(x y)^{2}\left(\begin{array}{l}
1 \\
0
\end{array}\right),
$$

$$
\left(\begin{array}{c}
0 \\
x y^{2}
\end{array}\right)=-\left(\begin{array}{c}
x^{2} y \\
-x y^{2}
\end{array}\right)+\left(\begin{array}{c}
x^{2} y \\
0
\end{array}\right),
$$

every vector field determined in part (ii) can be expressed in the form

$$
\alpha x^{2} y \frac{\partial}{\partial x}+X^{*}
$$

with $X^{*}$ as in (10). Thus, part (a) is proven.

(iv) Now let $X$ as in part (a). Routine computations show

$$
\begin{aligned}
L_{0}= & B(y-x)+\alpha x y+x y\left(y P_{0}+x Q_{0}\right), \\
L_{1}= & -\alpha(1+y)+C(y-x)-y(1+y) P_{0}-x(1+x) Q_{0}, \\
\operatorname{div} X= & 2 \alpha x y+\left(-x(x+1) B_{x}+y(y+1) B_{y}\right)+2 B(y-x)-x^{2} y C_{x}+ \\
& x y^{2} C_{y}+2 x y\left(y P_{0}+x Q_{0}\right)+(x y)^{2}\left(P_{0 x}+Q_{0 y}\right) .
\end{aligned}
$$

Note that $L_{1}$ is the cofactor of $F=\exp \left(f_{1} / f_{0}\right)$. The integrating factor condition, according to Theorem 4 , is

$$
\lambda_{0} L_{0}+\lambda_{1} L_{1}=\operatorname{div} X
$$


Evaluation at $x=y=0$ yields $\lambda_{1} \alpha=0$. According to Lemma 11, or Theorem 12 applied to $f_{0}^{(1)}=x, f_{0}^{(2)}=y$, there exists a Darboux first integral. This proves part (b).

Note that the Darboux integral is not necessarily of the form determined in Theorem 12: Rather than powers of $f_{0}$ one has products of powers of its prime factors $x$ and $y$.

For our second example, we consider algebraic curves that intersect nontransversally.

Proposition 15. For $f_{0}=x^{2}-y$ and $f_{1}=y$ the following holds.

(a) A polynomial vector field $X=(P, Q)$ satisfies

$$
\begin{aligned}
& X\left(f_{0}\right)=L_{0} f_{0}, \\
& X\left(f_{1}\right)=L_{0} f_{1}+L_{1} f_{0},
\end{aligned}
$$

for suitable polynomials $L_{0}$ and $L_{1}$ if and only if

$$
\left(\begin{array}{c}
P \\
Q
\end{array}\right)=A\left(\begin{array}{c}
x \\
2 y
\end{array}\right)+\left(x^{2}-y\right) B\left(\begin{array}{c}
1 \\
2 x
\end{array}\right)+\left(x^{2}-y\right)^{2} C\left(\begin{array}{c}
0 \\
1
\end{array}\right) \text {, }
$$

with arbitrary polynomials $A, B$ and $C$.

(b) The particular vector fields of type

$$
\left(\begin{array}{c}
P \\
Q
\end{array}\right)=(\alpha x+\beta y)\left(\begin{array}{c}
x \\
2 y
\end{array}\right)+\left(x^{2}-y\right)\left(\begin{array}{c}
1 \\
2 x
\end{array}\right),
$$

where $\alpha, \beta \in \mathbb{C}, \alpha \neq 0$ and $\beta \neq 0$, admit the Darboux integrating factor $f_{0}^{-\frac{5}{2}} \exp \left(\frac{f_{1}}{f_{0}}\right)^{\frac{\alpha}{2}}$ but have no Darboux first integral.

Proof: (i) By Lemma 6(a) of [6] all polynomial vector fields having $f_{0}=0$ as invariant algebraic curve can be written as

$$
\left(\begin{array}{c}
P \\
Q
\end{array}\right)=\tilde{R}\left(\begin{array}{c}
1 \\
2 x
\end{array}\right)+\left(x^{2}-y\right)\left(\begin{array}{c}
\tilde{P}_{0} \\
\tilde{Q}_{0}
\end{array}\right) .
$$

The stationarity of $(0,0)$ (note $\left\{f_{0}=0\right\} \cap\left\{f_{1}=0\right\}=\{(0,0)\}$ ) forces that $\tilde{R}=R_{1} x+R_{2} y$, and

$$
\begin{aligned}
\left(\begin{array}{c}
P \\
Q
\end{array}\right)= & R_{1}\left(\begin{array}{c}
x \\
2 x^{2}
\end{array}\right)+R_{2}\left(\begin{array}{c}
y \\
2 x y
\end{array}\right)+\left(x^{2}-y\right)\left(\begin{array}{c}
\tilde{P}_{0} \\
\tilde{Q}_{0}
\end{array}\right) \\
= & R_{1}\left(\begin{array}{c}
x \\
2 y
\end{array}\right)+R_{2} x\left(\begin{array}{c}
x \\
2 y
\end{array}\right)+ \\
& +\left(x^{2}-y\right)\left[\left(\begin{array}{c}
\tilde{P}_{0} \\
\tilde{Q}_{0}
\end{array}\right)+R_{1}\left(\begin{array}{c}
0 \\
2
\end{array}\right)+R_{2}\left(\begin{array}{c}
-1 \\
0
\end{array}\right)\right] \\
= & A\left(\begin{array}{c}
x \\
2 y
\end{array}\right)+\left(x^{2}-y\right)\left(\begin{array}{c}
B \\
Q_{0}
\end{array}\right) .
\end{aligned}
$$


(ii) The cofactor of $f_{0}$ equals $L_{0}=2 A+2 x B-Q_{0}$, and the condition $X\left(f_{1}\right)-L_{0} f_{1} \in\left\langle f_{0}\right\rangle$ is equivalent to

$$
\left(x^{2}-y\right) Q_{0}-\left(2 x B-Q_{0}\right) y \in\left\langle f_{0}\right\rangle,
$$

which gives $2 x B-Q_{0} \in\left\langle f_{0}\right\rangle$, and so $Q_{0}=2 x B+C f_{0}$. Substituting this expression in the vector field $X$ yields part (a).

(iii) For a vector field as in part (a), the algebraic curve $f_{0}=0$ is invariant and has cofactor $L_{0}=2 A-f_{0} C$. The exponential factor $\exp \left(f_{1} / f_{0}\right)$ is also invariant and has cofactor $L_{1}=x(2 B+C x)$. In particular, for $A=\alpha x+\beta y$, $B=1$ and $C=0$ we have $L_{0}=2 \alpha x+2 \beta y$ and $L_{1}=2 x$. The divergence of the vector field is equal to $4 \alpha x+5 \beta y$. Therefore, the relation

$$
-\frac{5}{2} L_{0}+\frac{\alpha}{2} L_{1}=-\operatorname{div}(X)
$$

holds, and by Theorem 4 the vector field $X$ has the Darboux integrating factor $f_{0}^{-\frac{5}{2}} f_{1}^{\frac{\alpha}{2}}$. The relation $\lambda_{0} L_{0}+\lambda_{1} L_{1}=0$ for $\beta \neq 0$ gives $\lambda_{0}=\lambda_{1}=0$. Hence, $X$ cannot have a Darboux first integral formed only by the polynomial $f_{0}$ and the exponential factor $\exp \left(f_{1} / f_{0}\right)$. To prove that $X$ cannot admit any Darboux first integral, we need two more steps.

(iv) First we claim that $\left\{f_{0}=0\right\}$ is the only invariant algebraic curve admitted by $X$. As a consequence, the only candidates for Darboux first integrals are of type $f_{0}^{\theta} \cdot \exp \left(g / f_{0}^{n}\right)$ with a polynomial $g$, a nonnegative integer $n$ and some complex $\theta$. As a second consequence, the vector field admits no polynomial first integral. To prove this claim we consider stationary points at infinity; see Perko [11] and the computations in [16].

The leading term of $X$ is $2 x^{3} \cdot \partial / \partial y$, hence the stationary points at infinity are determined by the prime factors of the homogeneous polynomial

$$
\operatorname{det}\left(\begin{array}{cc}
x & 0 \\
y & 2 x^{3}
\end{array}\right)=2 x^{4}
$$

Thus there is only one stationary point at infinity, viz. $(0: 1: 0)$. We determine the corresponding Poincaré transform by the procedure outlined in $[16], \S 3$. First homogenize the vector field to obtain

$$
g=\left(\begin{array}{c}
g_{1} \\
g_{2} \\
0
\end{array}\right)=\left(\begin{array}{c}
-y z^{2}+(1+\alpha) x^{2} z+\beta x y z \\
(2 \alpha-2) x y z+2 \beta y^{2} z+2 x^{3} \\
0
\end{array}\right),
$$


then take the projection with respect to $y$, i.e.,

$$
\begin{aligned}
\tilde{g}=\left(\begin{array}{c}
\tilde{g}_{1} \\
0 \\
\tilde{g}_{3}
\end{array}\right) & =-g_{2} \cdot\left(\begin{array}{c}
x \\
y \\
z
\end{array}\right)+y \cdot g \\
& =\left(\begin{array}{c}
(3-\alpha) x^{2} y z-\beta x y^{2} z-2 x^{4}-y^{2} z^{2} \\
0 \\
-2 \beta y^{2} z^{2}-(2 \alpha-2) x y z^{2}-2 x^{3} z
\end{array}\right) .
\end{aligned}
$$

We obtain the Poincaré transform

$$
\left(\begin{array}{c}
P^{*}(x, z) \\
Q^{*}(x, z)
\end{array}\right):=\left(\begin{array}{c}
\tilde{g}_{1}(x, 1, z) \\
\tilde{g}_{3}(x, 1, z)
\end{array}\right)=\left(\begin{array}{c}
-\beta x z-z^{2}+(3-\alpha) x^{2} z-2 x^{4} \\
-2 \beta z^{2}-(2 \alpha-2) x z^{2}-2 x^{3} z
\end{array}\right)
$$

and the stationary point of $X$ at infinity corresponds to the stationary point 0 for this system.

This stationary point is not elementary, therefore we use a blow-up (see e.g. Arnold [1], Ch. 1, $\S 2$, where this is called a $\sigma$-process) to obtain the vector field

$$
\begin{aligned}
z \cdot Y & :=\frac{1}{z} \cdot\left(\begin{array}{c}
P^{*}(x z, z)-x Q^{*}(x z, z) \\
z Q^{*}(x z, z)
\end{array}\right) \\
& =z \cdot\left(\begin{array}{c}
-1+\beta x+(1+\alpha) x^{2} z-4 x^{4} z^{2} \\
-2 \beta z-(2 \alpha-2) x z^{2}-2 x^{3} z^{3}
\end{array}\right) .
\end{aligned}
$$

The invariant line $\{z=0\}$ of $Y$ is the blow-up of $(0,0)$, hence we investigate the stationary points on this line. Obviously, $\left(\beta^{-1}, 0\right)$ is the only such stationary point, and its Jacobian is easily computed as

$$
\left(\begin{array}{cc}
\beta & * \\
0 & -2 \beta
\end{array}\right)
$$

The eigenvalue ratio equals -2 , and thus, e.g. by [16], Theorem 2.3 there exist exactly two irreducible local analytic invariant curves containing this point. Blowing down again, we see that the stationary point 0 of the Poincaré transform is contained in exactly two local analytic invariant curves, and one of these is the line at infinity. But every invariant algebraic curve induces at least one irreducible local invariant curve for some stationary point at infinity. Therefore, apart from $\left\{f_{0}=0\right\}$ there can be no others.

(v) To preclude the existence of a Darboux first integral $f_{0}^{\mu} \cdot \exp (g)$, note that the cofactor of such an expression is equal to

$$
K:=\mu \cdot L_{0}+X(g)=\mu \cdot L_{0}+P \cdot g_{x}+Q \cdot g_{y} .
$$

The assumption $K=0$ implies $K(x, 0)=0$ in particular, and evaluation yields

$$
0=2 \alpha \mu \cdot x+x^{2} \cdot(\cdots)
$$


and thus $\mu=0$. Since $X$ has no polynomial first integral, this is impossible. (vi) To preclude the existence of a Darboux first integral $f_{0}^{\mu} \cdot \exp \left(g / f_{0}^{n}\right)$ with some integer $n>1$, we will show that $\left\{f_{0}=0\right\}$ has exact multiplicity 2. Assume that there are polynomials $f_{2}$ and $L_{2}$ such that

$$
X\left(f_{2}\right)=L_{0} f_{2}+L_{1} f_{1}+L_{2} f_{0} .
$$

Consider the degree function deg defined by $\operatorname{deg}(x)=1$ and $\operatorname{deg}(y)=2$. Then $f_{0}$ and $f_{1}$ are homogeneous of degree 2 with respect to deg, and $L_{1}$ is homogeneous of degree 1 . The vector fields

$$
X_{1}:=\beta y \cdot\left(\begin{array}{c}
x \\
2 y
\end{array}\right), \quad X_{2}:=\alpha x \cdot\left(\begin{array}{c}
x \\
2 y
\end{array}\right)+\left(x^{2}-y\right) \cdot\left(\begin{array}{c}
1 \\
2 x
\end{array}\right),
$$

induce deg-homogeneous derivations of respective degrees 3 and 2; thus $\operatorname{deg}\left(X_{1}(g)\right)=\operatorname{deg}(g)+2$ and $\operatorname{deg}\left(X_{2}(g)\right)=\operatorname{deg}(g)+1$ for all homogeneous $g$. Moreover $X=X_{1}+X_{2}$. We will write a polynomial

$$
g=g^{(0)}+g^{(1)}+\cdots+g^{(r)},
$$

as the sum of its deg-homogeneous terms; in particular $L_{0}=L_{0}^{(1)}+L_{0}^{(2)}$ with $L_{0}^{(1)}=2 \alpha x$ and $L_{0}^{(2)}=2 \beta y$. With $f_{2}=f_{2}^{(0)}+\cdots$ and $L_{2}=L_{2}^{(0)}+\cdots$ compare terms of smallest degree in (32). At degree 1 one has

$$
0=L_{0}^{(1)} f_{2}^{(0)}
$$

and therefore $f_{2}^{(0)}=0$. Using this and $f_{2}^{(1)}=\mu x$ for some $\mu$, the degree 2 terms yield

$$
\begin{aligned}
X_{2}\left(f_{2}^{(1)}\right) & =L_{0}^{(1)} f_{2}^{(1)}+\left(x^{2}-y\right) \cdot L_{2}^{(0)}, \quad \text { or } \\
\alpha \mu x^{2}+\mu\left(x^{2}-y\right) & =2 \alpha \mu x^{2}+L_{2}^{(0)}\left(x^{2}-y\right),
\end{aligned}
$$

which forces $\mu=L_{2}^{(0)}=0$ in view of $\alpha \neq 0$. Using $f_{2}^{(1)}=0$ at degree 3 , we obtain

$$
X_{2}\left(f_{2}^{(2)}\right)=2 \alpha x f_{2}^{(2)}+2 x y+\left(x^{2}-y\right) \cdot L_{2}^{(1)} .
$$

Since the terms $2 \alpha x f_{2}^{(2)}$ on the left and right hand sides cancel, we obtain the contradiction that $x^{2}-y$ divides $2 x y$. Thus, relation (32) is impossible.

As a final example we discuss a system admitting an invariant curve defined by $f=y^{2}+x^{3}$. Since the curve $\{f=0\}$ has a cusp at the origin, it does not satisfy the criteria in Theorem 10. The form of the first integral follows the pattern for these exceptional cases conjectured by Żoładek [17], where more examples are given. 
Proposition 16. The system

$$
\dot{x}=\frac{\alpha}{3} y+2 \beta x, \quad \dot{y}=3 \beta y-\frac{\alpha}{2} x^{2},
$$

admits an inverse integrating factor $\left(y^{2}+x^{3}\right)^{5 / 6}$, and a first integral

$$
\phi=\alpha\left(y^{2}+x^{3}\right)^{1 / 6}+\beta \int^{y^{2} / x^{3}} \frac{d z}{(1+z)^{5 / 6} z^{1 / 2}} .
$$

But the system does not admit a Darboux first integral if $\alpha \neq 0$ and $\beta \neq 0$.

Proof. Let $X$ be the vector field corresponding to the system. Note that $X(f)=6 \beta f$.

(i) Assume that $X$ admits the polynomial $g$, so that $X(g)=L \cdot g$ for some polynomial $L$, and $g$ is not a multiple of $f$. Comparing homogeneous terms of highest degree shows that the highest order terms of $g$ are a multiple of $x^{n}$ for some $n$. The next highest terms show that $L$ is a constant. There is a unique representation

$$
g=A(x)+y \cdot B(x)+f \cdot \tilde{g}
$$

and one obtains the corresponding representation

$$
\begin{aligned}
X(g)= & \left(2 \beta x+\frac{\alpha}{3} y\right) \cdot\left(A^{\prime}(x)+y B^{\prime}(x)\right)+\left(3 \beta y-\frac{\alpha}{2} x^{2}\right) \cdot B(x)+f \cdot h \\
= & 2 \beta x A^{\prime}(x)-\frac{\alpha}{2} x^{2} B(x)-\frac{\alpha}{3} x^{3} B^{\prime}(x) \\
& \quad+y \cdot\left(2 \beta x B^{\prime}(x)+\frac{\alpha}{3} A^{\prime}(x)+3 \beta B(x)\right)+f \cdot \tilde{h}
\end{aligned}
$$

for some polynomials $h$ and $\tilde{h}$, since $y^{2} \equiv x^{3} \bmod f$. Evaluating $X(g)=L \cdot g$ for some constant $L$ yields

$$
\begin{aligned}
2 \beta A^{\prime}-\frac{\alpha}{2} x^{2} B-\frac{\alpha}{3} x^{3} B^{\prime} & =L \cdot A ; \\
2 \beta x B^{\prime}+\frac{\alpha}{3} A^{\prime}+3 \beta B & =L \cdot B .
\end{aligned}
$$

Assuming that $B \neq 0$, with $\delta B=m$, the second equation shows that $\delta A \leq 1+m$. Comparing terms of highest degree in the first equation then yields $\frac{1}{2} x^{m+2}+\frac{m}{3} x^{m+2}=0$; this contradiction forces $B=A=0$. But this means that $g$ is a multiple of $f$, contrary to our assumption. In particular there is no polynomial first integral.

(ii) The only possible exponential factors are therefore of the form $\exp \left(g / f^{n}\right)$ for some $n$. There is one obvious such factor: $\exp (x)$ with cofactor $\frac{\alpha}{3} y+2 \beta x$. We shall show that there are no others. We first exclude the case $n=0$. For, suppose that there is an exponential factor of $X$ of the form $\exp (g)$ for some polynomial $g$. This would satisfy $X(g)=L$ for some polynomial $L$ of degree 1 . Replacing $g$ by $g-k x$ for some suitably chosen constant $k$ we can 
assume that $\exp (g)$ now has a cofactor of the form $a x+b$, for some constants $a$ and $b$. A calculation along the lines of part (i) is performed giving (34)and (35) with $L \cdot A$ and $L \cdot B$ replaced by $a x+b$ and 0 respectively. As above, there are no nontrivial solutions and so $g=f \tilde{g}$ for some polynomial $\tilde{g}$. But then $X(g)=f(6 \beta g+X(g))=a x+b$ which is not possible unless $a=b=0$, but this implies that there is a polynomial first integral which we excluded above.

(iii) Finally we exclude exponential factors of the form $\exp \left(g / f^{n}\right)$ for some $n>1$ where we assume $f$ does not divide $g$. We therefore have $X(g)-$ $6 \beta n g=f^{n} \tilde{L}$ for some cofactor $L$. Exactly as in part (i) we obtain (34) and (35) with $L \cdot A$ and $L \cdot B$ replaced by $6 \beta n A$ and $6 \beta n B$ respectively and again find that $g$ must be a multiple of $f$, which we have excluded. Thus, the only exponential factors or invariant algebraic curves supported by $X$ from which we can construct our Darboux first integral is $f$ and $\exp (x)$. However such a construction is not possible as their cofactors are independent over $\mathbb{C}$.

\section{REFERENCES}

[1] V.I. ARnold, Geometrical methods in the theory of ordinary differential equations, 2nd ed., Springer-Verlag Berlin/New York, 1988.

[2] C.J. Christopher, Invariant algebraic curves and conditions for a center, Proc. Roy. Soc. Edinburgh 124A (1994), 1209-1229.

[3] C.J. Christopher, Liouvillian first integrals of second order polynomial differential equations, Electron. J. Differential Equations 49 (1999), 7 pp. (electronic).

[4] C. Christopher and J. Llibre, Algebraic Aspects of Integrability for Polynomial Systems, Qualitative Theory of Dynamical Systems 1 (1999), 71-95.

[5] C. Christopher And J. Llibre, Integrability via Invariant Algebraic Curves for Planar Polynomial Differential Systems, Annals of Differential Equations 16 (2000), 5-19.

[6] C. J. Christopher, J. Llibre, C. Pantazi and X. Ziang, Darboux integrability and invariant algebraic curves for planar polynomial systems, J. Physics A: Gen. Math. 35 (2002), 2457-2476.

[7] C. Christopher, J. Llibre and V. Pereira, Multiplicity of invariant algebraic curves in polynomial vector fields, preprint 2004.

[8] G. Darboux, Mémoire sur les équations différentielles algébriques du premier ordre et du premier degré (Mélanges), Bull. Sci. Math. 2ème série 2 (1878), 60-96; 123144; 151-200.

[9] H. Giacomini, J. Llibre and M. Viano, On the nonexistence, existence and uniqueness of limit cycles, Nonlinearity 9 (1996), 501-516.

[10] J. Llibre And C. PANtazi, Polynomial differential systems having a given Darbouxian first integral, Bull. Sci. Math. 188 (2004), 775-788.

[11] L. Perko, Differential equations and dynamical systems, 2nd ed., Springer-Verlag, Berlin/New York (1996).

[12] M.J. Prelle AND M.F. Singer, Elementary first integrals of differential equations, Trans. Amer. Math. Soc. 279 (1983), 215-229. 
[13] I. R. Shafarevich, Basic algebraic geometry, Springer-Verlag, Berlin/New York (1977).

[14] M.F. Singer, Liouvillian first integrals of differential equations, Trans. Amer. Math. Soc. 333 (1992), 673-688.

[15] S. WALChER, Plane polynomial vector fields with prescribed invariant curves, Proc. Roy. Soc. Edinburgh 130A (2000), 633-649.

[16] S. Walcher, On the Poincaré problem, J. Differential Eqs. 166 (2000), 51-78.

[17] H. ŻoŁA̧DEK, The extended monodromy group and Liouvillian first integrals, J. Dynamical and Control Systems 4 (1998), 1-28.

1 Department of Mathematics and Statistics, University of Plymouth, PlyMOUTH PL2 3AJ, U.K.

E-mail address: c.christopher@plymouth.ac.uk

2 Departament de Matemàtiques, Universitat Autònoma de Barcelona, 08193 Bellaterra, Barcelona, Spain

E-mail address: jllibre@mat.uab.es

3 Departament de Matemàtica Aplicada I, Universitat Politècnica de Catalunya, ETSEIB, Av. Diagonal, 647, 08028 Barcelona, Spain

E-mail address: Chara.Pantazi@upc.edu

${ }^{4}$ Lehrstuhl A für Mathematik, RWTH Aachen, 52056 Aachen, Germany

E-mail address: walcher@mathA.rwth.aachen.de 